\title{
Type-X two-Higgs-doublet model in light of the muon $g-2$ : Confronting Higgs boson and collider data
}

\author{
Adil Jueid $\odot,{ }^{*}$ Jinheung Kim, ${ }^{\dagger}$ Soojin Lee, ${ }^{*}$ and Jeonghyeon Song $\odot^{\S}$ \\ Department of Physics, Konkuk University, Seoul 05029, Republic of Korea
}

(Received 12 July 2021; accepted 19 October 2021; published 11 November 2021)

\begin{abstract}
The recent Fermilab measurement of the muon anomalous magnetic moment yields $4.2 \sigma$ deviations from the SM prediction when combined with the BNL E821 experiment results. In the type-X twoHiggs-doublet model, we study the consequence of imposing the observed muon $g-2$, along with the constraints from theoretical stabilities, electroweak oblique parameters, Higgs precision data, and direct searches. For a comprehensive study, we scan the whole parameter space in two scenarios, the normal scenario where $h_{\mathrm{SM}}=h$ and the inverted scenario where $h_{\mathrm{SM}}=H$, where $h(H)$ is the light (heavy) $C P$-even Higgs boson. We found that large $\tan \beta$ (above 100) and light pseudoscalar mass $M_{A}$ are required to explain the muon $g-2$ anomaly. This breaks the theoretical stability unless the scalar masses satisfy $M_{A}^{2} \simeq M_{H^{ \pm}}^{2} \simeq m_{12}^{2} \tan \beta \approx M_{H / h}^{2}$. The direct search bounds at the LEP and LHC exclude the light $A$ window with $M_{A} \lesssim 62.5 \mathrm{GeV}$. We also show that the observed electron anomalous magnetic moment is consistent with the model prediction, but the lepton flavor universality data in the $\tau$ and $Z$ decays are not. For a separate exploration of the model, we propose the golden mode $p p \rightarrow A h / A H \rightarrow 4 \tau$ at the HL-LHC.
\end{abstract}

DOI: 10.1103/PhysRevD.104.095008

\section{INTODUCTION}

The recent measurement of the muon anomalous magnetic moment by the Fermilab National Accelerator Laboratory (FNAL) Muon $g-2$ experiment [1,2] achieved unprecedented precision. When combined with the old result of the Brookhaven National Laboratory (BNL) E821 measurement [3], it reads as

$$
a_{\mu}^{\exp }=116592061(41) \times 10^{-11},
$$

where $a_{\mu}=(g-2)_{\mu} / 2$. As the experimental error is becoming comparable with the theoretical error, ${ }^{1}$ reliable and accurate calculation of the SM prediction is more important than ever. The recent progress includes five loops in QED [4] and two loops in electroweak interactions [5,6]. Nevertheless, the most dominant contribution is from the strong interaction dynamics at $\mathcal{O}(1) \mathrm{GeV}$, which is

*adiljueid@konkuk.ac.kr

jinheung.kim1216@gmail.com

soojinlee957@gmail.com

\$ihsong@konkuk.ac.kr

${ }^{1}$ The experimental measurement will be improved in a short timescale. For instance, FNAL will provide a new measurement of $a_{\mu}$ in the summer of 2022 after including more datasets.

Published by the American Physical Society under the terms of the Creative Commons Attribution 4.0 International license. Further distribution of this work must maintain attribution to the author(s) and the published article's title, journal citation, and DOI. Funded by SCOAP ${ }^{3}$. categorized into the hadronic vacuum polarization (HVP) [7-14] and the hadronic light-by-light (HLbL) scattering [15-27]. These QCD corrections cannot be computed using perturbation theory. We have to resort to nonperturbative methods, either lattice QCD or data-driven methods. On the lattice side, the recent calculation of the leading order HVP (LO-HVP) contributions to $a_{\mu}$ by the Budapest-Marseille-Wuppertal collaboration [28] yields $\left.a_{\mu}\right|_{\text {LO-HVP }}=707.5(5.3) \times 10^{-10}$. If we take this result at face value, the Fermilab measurement of $a_{\mu}$ is consistent with the SM prediction at $\sim 2 \sigma$. On the datadriven method side, however, the calculation of the HVP contribution $[9,11,12]$ supports the longstanding discrepancy between the muon $g-2$ experiment and the SM prediction, as

$$
\Delta a_{\mu}^{\mathrm{obs}}=a_{\mu}^{\mathrm{exp}}-a_{\mu}^{\mathrm{SM}}=251(59) \times 10^{-11} .
$$

Which method is more appropriate needs further investigation. One checking point is the connection of the QCD corrections to electroweak precision fits [29-32], since some of the most important inputs to HVP and HLbL contributions come from measuring the $R(s)$-ratio in $e^{+} e^{-}$ collisions. Lately, some tension was reported between the lattice result and the electroweak data [30,31]. Another critical topic is how to combine the probability distribution functions with different errors.

In this paper, we take the $4.2 \sigma$ deviation in Eq. (2), which calls for new physics (NP) explanation. In a short time, 
various NP models have been vigorously studied for the muon $g-2$, focusing on a supersymmetric theory [33-45], leptophilic boson model [46,47], singlet scalar model [48], three Higgs doublet model [49], leptoquark model [50,51], $L_{\mu}-L_{\tau}$ model [52,53], $B-L$ or $B-3 L$ gauge model [54,55], flavorful scalar model [56], seesaw model [57], simplified model with minimal field contents [58], effective field theory [59], axion model [60,61], two-Higgs-doublet model (2HDM) [62-71], or 2HDM with a singlet scalar model $[72,73]$. These efforts shall continue because each NP model as a solution for the observed $\Delta a_{\mu}$ should simultaneously explain a vast amount of experimental data in particle physics.

From this motivation, we study the $C P$ invariant type- $\mathrm{X}$ (lepton-specific) $2 \mathrm{HDM}$ in light of the muon $g-2$. In type$X$, the couplings of the new scalar bosons to the SM quarks are inversely proportional to $\tan \beta$, the ratio of two vacuum expectation values of two Higgs doublet fields, but those to the charged leptons are linearly proportional to $\tan \beta$. Large $\tan \beta$ can enhance the new contributions of extra Higgs bosons to $\Delta a_{\mu}$, while suppressing the contributions to the hadron-related data such as $B \rightarrow K \mu^{+} \mu^{-}$and $B_{s} \rightarrow \mu^{+} \mu^{-}$ [74]. Since the other three types (type-I, type-II, and type$\mathrm{Y})$ cannot accommodate this feature, there have been extensive studies of type- $\mathrm{X}$ for the muon anomalous magnetic moment [75-82].

A comprehensive study of type- $\mathrm{X}$ for $\Delta a_{\mu}$, including the LHC Run-1 and LEP results as well as the lepton flavor universality (LFU) data in the $Z$ and $\tau$ decays, was first conducted in Ref. [78]. Partial updates have followed, focusing on the LFU data [79] or the LHC data [80-82]. We generalize the previous studies of the type-X 2HDM both in theoretical setup and in data analysis. First, we take the general setting in the Higgs sector, by considering two scenarios, the "normal" scenario where the observed Higgs boson is the lighter $C P$-even scalar $h$ and the "inverted" scenario where the heavier $C P$-even scalar $H$ is the observed one. The inverted scenario with a new light $C P$-even scalar has recently drawn a lot of interest because of the $3 \sigma$ excess in the diphoton invariant mass distribution at around $96 \mathrm{GeV}$ [83], but has not been analyzed in the context of the muon $g-2$. This scenario seems incompatible with the recent measurement of positive $\Delta a_{\mu}$, because the dominant Barr-Zee contributions of the $\tau^{ \pm}$loop mediated by the light $C P$-even $h$ are negative $[84,85]$. We need to answer whether the inverted scenario remains viable.

For the general data analysis, we will investigate all the latest data of the LHC Run-2, the electron anomalous magnetic moment [86,87], the LFU data (adopting the updated HFLAV global fit results [88] and Michel parameters [89] in the $\tau$ decay), as well as theoretical stabilities and electroweak oblique parameters. We will also include the correlations among the observables. The correlations are often neglected in the literature, but they play a vital role in constraining new physics models. To draw a general conclusion on the type-X 2HDM, we will scan the whole parameter space without any extra assumption on the masses or the couplings. Furthermore, the tension in the type-X when simultaneously explaining $\Delta a_{\mu}$ and LFU data shall be quantified through the global $\chi^{2}$ fit. Finally, the customized search strategy for the viable parameter space at the HL-LHC is to be studied. These are our contributions to the phenomenology of the type-X $2 \mathrm{HDM}$ in light of the new Fermilab measurement of $\Delta a_{\mu}$.

The paper is organized in the following way. In Sec. II, we briefly review the type-X $2 \mathrm{HDM}$ and describe the characteristics of the normal and inverted scenarios in the Higgs alignment limit. In Sec. III, we discuss the new contributions of the type-X 2HDM to $\Delta a_{\mu}$. Section IV describes our scanning strategies in three steps and shows the results of the allowed parameter space at each step. Section $\mathrm{V}$ deals with the electron anomalous magnetic moment and the LHC signatures. In Sec. VI, we check the consistency of the model with the LFU data in the $\tau$ and $Z$ decays. Conclusions are given in Sec. VII.

\section{TYPE-X 2HDM}

The 2HDM accommodates two complex $S U(2)_{L}$ Higgs doublet scalar fields, $\Phi_{1}$ and $\Phi_{2}$ [90]:

$$
\Phi_{i}=\left(\begin{array}{c}
w_{i}^{+} \\
\frac{v_{i}+h_{i}+i \eta_{i}}{\sqrt{2}}
\end{array}\right), \quad i=1,2,
$$

where $v=\sqrt{v_{1}^{2}+v_{2}^{2}}=246 \mathrm{GeV}$. Using the simplified notation of $s_{x}=\sin x, c_{x}=\cos x$, and $t_{x}=\tan x$, we define $t_{\beta}=v_{2} / v_{1}$. To prevent the tree-level flavor changing neutral currents, a discrete $Z_{2}$ symmetry is imposed as $\Phi_{1} \rightarrow \Phi_{1}$ and $\Phi_{2} \rightarrow-\Phi_{2}$ [91,92]. The most general, renormalizable, and $C P$ conserving scalar potential with softly broken $Z_{2}$ symmetry is

$$
\begin{aligned}
V_{\Phi}= & m_{11}^{2} \Phi_{1}^{\dagger} \Phi_{1}+m_{22}^{2} \Phi_{2}^{\dagger} \Phi_{2}-m_{12}^{2}\left(\Phi_{1}^{\dagger} \Phi_{2}+\text { H.c. }\right) \\
& +\frac{1}{2} \lambda_{1}\left(\Phi_{1}^{\dagger} \Phi_{1}\right)^{2}+\frac{1}{2} \lambda_{2}\left(\Phi_{2}^{\dagger} \Phi_{2}\right)^{2}+\lambda_{3}\left(\Phi_{1}^{\dagger} \Phi_{1}\right)\left(\Phi_{2}^{\dagger} \Phi_{2}\right) \\
& +\lambda_{4}\left(\Phi_{1}^{\dagger} \Phi_{2}\right)\left(\Phi_{2}^{\dagger} \Phi_{1}\right)+\frac{1}{2} \lambda_{5}\left[\left(\Phi_{1}^{\dagger} \Phi_{2}\right)^{2}+\text { H.c. }\right],
\end{aligned}
$$

where the $m_{12}^{2}$ term softly breaks the $Z_{2}$ parity. There are five physical Higgs bosons, the light $C P$-even scalar $h$, the heavy $C P$-even scalar $H$, the $C P$-odd pseudoscalar $A$, and two charged Higgs bosons $H^{ \pm}$. The relations of the physical Higgs bosons with the weak eigenstates in Eq. (3) via two mixing angles $\alpha$ and $\beta$ are referred to Ref. [93,94]. Note that the SM Higgs boson is a linear combination of $h$ and $H$, as

$$
h_{\mathrm{SM}}=s_{\beta-\alpha} h+c_{\beta-\alpha} H .
$$


The Yukawa couplings to the SM fermions are written by

$$
\begin{aligned}
\mathcal{L}_{\text {Yuk }}= & -\sum_{f}\left(\frac{m_{f}}{v} y_{f}^{h} \bar{f} f h+\frac{m_{f}}{v} y_{f}^{H} \bar{f} f H-i \frac{m_{f}}{v} y_{f}^{A} \bar{f} \gamma_{5} f A\right) \\
& -\left\{\frac{\sqrt{2}}{v} \bar{t}\left(m_{t} y_{t}^{A} P_{L}+m_{b} y_{b}^{A} P_{R}\right) b H^{+}\right. \\
& \left.+\frac{\sqrt{2} m_{\ell}}{v} y_{\ell}^{A} \bar{\nu}_{\ell} P_{R} \ell H^{+}+\text {H.c. }\right\}
\end{aligned}
$$

where $P_{R, L}=\left(1 \pm \gamma^{5}\right) / 2$ and $\ell=\mu, \tau$.

The observed Higgs boson at a mass of $125 \mathrm{GeV}$ is similar to the SM Higgs boson, more strongly in type- $\mathrm{X}$ with large $t_{\beta}$ [95]. Therefore, we take the Higgs alignment limit where one of the $C P$-even neutral Higgs bosons is the SM Higgs boson $h_{\mathrm{SM}}$ [96-100]. There are two ways to realize the Higgs alignment limit, the "normal" and "inverted" scenarios. In the normal scenario, the observed Higgs boson is the lighter $C P$-even scalar $h$, i.e., $s_{\beta-\alpha}=1$. In the inverted scenario, $c_{\beta-\alpha}=1$ so that the heavier $C P$ even scalar $H$ is observed while the lighter one is hidden $[99,101]$. The model has five independent parameters in the physical basis,

$$
\left\{m_{\varphi^{0}}, M_{A}, M_{H^{ \pm}}, M^{2}, t_{\beta}\right\},
$$

where $M^{2}=m_{12}^{2} /\left(s_{\beta} c_{\beta}\right)$ and $\varphi^{0}$ is the new $C P$-even neutral Higgs boson, i.e., $\varphi^{0}=H$ in the normal scenario and $\varphi^{0}=h$ in the inverted scenario. The two scenarios are summarized as follows:

\begin{tabular}{c|c} 
normal scenario(NS) & inverted scenario(IS) \\
\hline$h_{\mathrm{SM}}=h, \varphi^{0}=H$ & $h_{\mathrm{SM}}=H, \varphi^{0}=h$ \\
$y_{f}^{h_{\mathrm{S}}}=1, s_{\beta-\alpha}=1$ & $y_{f}^{h_{\mathrm{SM}}}=1, c_{\beta-\alpha}=1$ \\
$y_{t}^{A}=-y_{t}^{\varphi^{0}}=\frac{1}{t_{\beta}}, y_{\ell}^{A}=y_{\ell}^{\varphi^{0}}=t_{\beta}$ & $y_{t}^{A}=y_{t}^{\varphi^{0}}=\frac{1}{t_{\beta}}, y_{\ell}^{A}=-y_{\ell}^{\varphi^{0}}=t_{\beta}$
\end{tabular}
[100]

In the Higgs alignment limit, the quartic couplings are

$$
\begin{aligned}
& \lambda_{1}=\frac{1}{v^{2}}\left[m_{125}^{2}+t_{\beta}^{2}\left(m_{\varphi^{0}}^{2}-M^{2}\right)\right], \\
& \lambda_{2}=\frac{1}{v^{2}}\left[m_{125}^{2}+\frac{1}{t_{\beta}^{2}}\left(m_{\varphi^{0}}^{2}-M^{2}\right)\right], \\
& \lambda_{3}=\frac{1}{v^{2}}\left[m_{125}^{2}-m_{\varphi^{0}}^{2}-M^{2}+2 M_{H^{ \pm}}^{2}\right] \\
& \lambda_{4}=\frac{1}{v^{2}}\left[M^{2}+M_{A}^{2}-2 M_{H^{ \pm}}^{2}\right] \\
& \lambda_{5}=\frac{1}{v^{2}}\left[M^{2}-M_{A}^{2}\right]
\end{aligned}
$$

where $m_{125}=125 \mathrm{GeV}$. As shall be shown in the next section, the observed $\Delta a_{\mu}$ requires large $t_{\beta}$. Then, the $t_{\beta}^{2}$ terms in $\lambda_{1}$ easily break the perturbativity of $\lambda_{1}$ unless $m_{\varphi^{0}}^{2}$ is extremely close to $M^{2}$, which is to be denoted by $m_{\varphi^{0}}^{2} \approx M^{2}$. When applying this approximate equality to the perturbativity of $\lambda_{3}$, we should accommodate quasidegeneracy between $M^{2}$ and $M_{H^{ \pm}}^{2}$. The mass degeneracy is weaker because of the absence of $t_{\beta}^{2}$ terms in $\lambda_{3}$. We use the notation of $M^{2} \simeq M_{H^{ \pm}}^{2}$ for the weak equality. The perturbativity of $\lambda_{4}$ and $\lambda_{5}$ finally yields $M_{A} \simeq M_{H^{ \pm}}$. In summary, the perturbativity of the quartic couplings for large $t_{\beta}$ limits the masses as

$$
M_{A} \simeq M_{H^{ \pm}} \simeq M \approx m_{\varphi^{0}} .
$$

For light $M_{A}$, the exotic Higgs decay of $h_{\mathrm{SM}} \rightarrow A A$ severely restricts the model. When writing $\mathcal{L}=$ $(1 / 2) \lambda_{A A}^{h_{\mathrm{SM}}} h_{\mathrm{SM}} A A$, the vertex is

$$
\lambda_{A A}^{h_{\mathrm{SM}}}=\frac{1}{v}\left(-m_{125}^{2}-2 M_{A}^{2}+2 M^{2}\right) .
$$

Because of the condition in Eq. (10), it is difficult to accommodate $\lambda_{A A}^{h_{S M}}=0 .{ }^{2}$ Since the Higgs precision measurement puts a strong bound on the exotic Higgs decay as $\mathcal{B}\left(h_{\mathrm{SM}} \rightarrow X X\right) \lesssim \mathcal{O}(0.1)$ [102], the parameter region with $M_{A} \leq m_{125} / 2$ is highly disfavored.

\section{III. $\Delta a_{\mu}$ IN THE TYPE-X 2HDM}

The type- $X$ 2HDM makes two kinds of new contributions to $\Delta a_{\mu}$, one-loop contributions and two-loop Barr-Zee contributions $[84,85]$. The one-loop contributions are mediated by $\varphi^{0}, A$, and $H^{ \pm}$, as [80]

$$
\begin{aligned}
\Delta a_{\mu}^{1-\text { loop }} & =\frac{G_{F} m_{\mu}^{2}}{4 \pi^{2} \sqrt{2}} \sum_{\phi}\left(y_{\mu}^{\phi}\right)^{2} \rho_{\phi}^{\mu} f_{\phi}\left(\rho_{\phi}^{\mu}\right) \\
& \simeq 2.6 \times 10^{-15} \sum_{\phi}\left(y_{\mu}^{\phi}\right)^{2}\left(\frac{100 \mathrm{GeV}}{M_{\phi}}\right)^{2} f_{\phi}\left(\rho_{\phi}^{\mu}\right),
\end{aligned}
$$

where $\phi=\left\{\varphi^{0}, A, H^{ \pm}\right\}, \rho_{j}^{i}=m_{i}^{2} / m_{j}^{2}$, and the expressions for the loop function $f_{\phi}$ are referred to Ref. [80]. The numerical factor in the second equality of Eq. (12) implies that the observed $\Delta a_{\mu}$ requires light $M_{\phi}$ and large $y_{\mu}^{\phi}$. Because $\rho_{\phi}^{\mu} \ll 1$, the loop functions show the following asymptotic behaviors:

\footnotetext{
${ }^{2}$ If the Higgs alignment is broken and $t_{\beta-\alpha}=\left(t_{\beta}-1 / t_{\beta}\right) \times$ $\left(M^{2}-m_{125}^{2}\right) /\left(2 M^{2}-2 M_{A}^{2}-m_{125}^{2}\right), \lambda_{A A}^{h_{\mathrm{SM}}}$ vanishes and the constraint from $h_{\mathrm{SM}} \rightarrow A A$ can be evaded. However, the equality involves five independent parameters of $\alpha, \beta, m_{125}, M^{2}$, and $M_{A}$, which is an unnatural fine-tuning without underlying symmetries.
} 

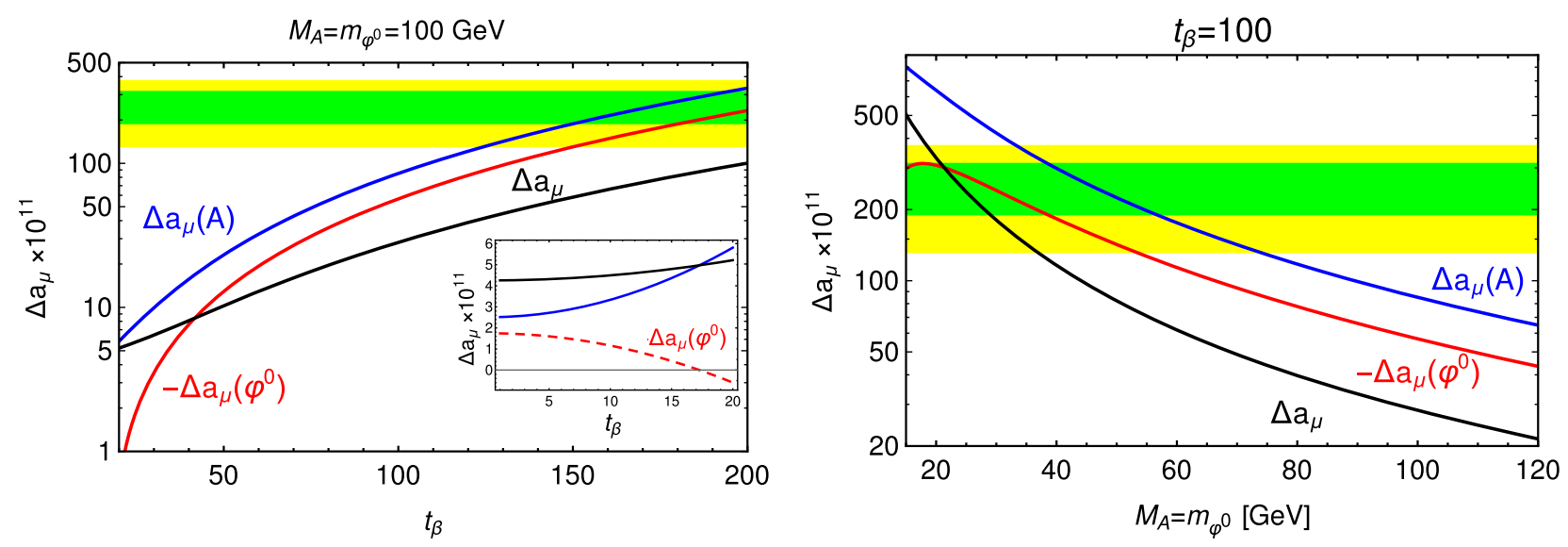

FIG. 1. $\Delta a_{\mu}$ from $A,-\Delta a_{\mu}$ from $\varphi^{0}$, and $\Delta a_{\mu}(A)+\Delta a_{\mu}\left(\varphi^{0}\right)$ as a function of $t_{\beta}$ with $M_{A}=m_{\varphi^{0}}=100 \mathrm{GeV}$ (left panel) and as a function of $M_{A}=m_{\varphi^{0}}$ for $t_{\beta}=100$ (right panel).

$$
\begin{aligned}
f_{\varphi^{0}}(\rho) & =-\ln \rho-7 / 6+\mathcal{O}(\rho), \\
f_{A}(\rho) & =+\ln \rho+11 / 6+\mathcal{O}(\rho), \\
f_{H^{ \pm}}(\rho) & =-1 / 6+\mathcal{O}(\rho) .
\end{aligned}
$$

It is clear to see that the one-loop contributions of the $C P$-even scalar $\varphi^{0}$ are positive while those of $A$ and $H^{ \pm}$are negative: $\Delta a_{\mu}^{1-\text { loop }}$ is proportional to the square of $y_{\mu}^{\phi}$.

More significant contributions to $\Delta a_{\mu}$ are from the twoloop Barr-Zee type diagrams with heavy fermions in the loop [84]:

$$
\Delta a_{\mu}^{\mathrm{BZ}}=\frac{G_{F} m_{\mu}^{2}}{4 \pi^{2} \sqrt{2}} \frac{\alpha_{\mathrm{em}}}{\pi} \sum_{f, \phi^{0}} N_{f}^{c} Q_{f}^{2} y_{\mu}^{\phi^{0}} y_{f}^{\phi^{0}} \rho_{\phi^{0}}^{f} g_{\phi^{0}}\left(\rho_{\phi^{0}}^{f}\right),
$$

where $f=t, b, \tau, \phi^{0}=\left\{\varphi^{0}, A\right\}, m_{f}, Q_{f}$ and $N_{f}^{c}$ are the mass, electric charge and color factor of the fermion $f$, and the loop functions are

$$
\begin{aligned}
g_{\varphi^{0}}(\rho) & =\int_{0}^{1} d x \frac{2 x(1-x)-1}{x(1-x)-\rho} \ln \frac{x(1-x)}{\rho} \\
g_{A}(\rho) & =\int_{0}^{1} d x \frac{1}{x(1-x)-\rho} \ln \frac{x(1-x)}{\rho} .
\end{aligned}
$$

For the top quark and $\tau^{ \pm}$loops, the factor $\rho_{\phi^{0}}^{f}$ in Eq. (14) significantly enhances $\Delta a_{\mu}^{\mathrm{BZ}}$ with respect to $\Delta a_{\mu}^{1-\text { loop }}$ in Eq. (12). The usual conclusion that a $C P$-even scalar boson makes a negative contribution to $\Delta a_{\mu}^{\mathrm{BZ}}$ holds true when $y_{\mu}^{\varphi^{0}} y_{f}^{\varphi^{0}}>0$. As shown in Eq. (8), the top quark incorporates $y_{\mu}^{\varphi^{0}} y_{t}^{\varphi^{0}}<0$ in both scenarios and thus generates positive two-loop Barr-Zee contributions.

In Fig. 1, we show $\Delta a_{\mu}(A)$ (blue line), $-\Delta a_{\mu}\left(\varphi^{0}\right)$ (red line), and $\Delta a_{\mu}(A)+\Delta a_{\mu}\left(\varphi^{0}\right)$ (black line) as a function of $t_{\beta}$ with $M_{A}=m_{\varphi^{0}}=100 \mathrm{GeV}$ in the left panel and as a function of $M_{A}=m_{\varphi^{0}}$ with $t_{\beta}=100$ in the right panel. To show negative $\Delta a_{\mu}\left(\varphi^{0}\right)$ in the logarithmic scale, we present $-\Delta a_{\mu}\left(\varphi^{0}\right)$. The horizontal green (yellow) area denotes the allowed region of $\Delta a_{\mu}$ at $1 \sigma(2 \sigma)$. The dominant contribution of $A$ is from two-loop Barr-Zee diagrams, which is always positive. The sign of the $\varphi^{0}$ contribution depends on the value of $t_{\beta}$. For very large $t_{\beta}, \Delta a_{\mu}\left(\varphi^{0}\right)$ is negative since the contribution of the $\tau^{ \pm}$loop in the twoloop Barr-Zee diagram is dominant. If $t_{\beta} \lesssim 17$, however, $\Delta a_{\mu}\left(\varphi^{0}\right)$ becomes positive (see the small figure inside the left panel) because dominant is the top quark loop in the two-loop Barr-Zee diagram. Although the contributions from both $A$ and $\varphi^{0}$ are positive for $t_{\beta} \lesssim 17$, the absolute value of $\Delta a_{\mu}$ is not large enough to explain $\Delta a_{\mu}^{\text {obs }}$. In the right panel, we show $\Delta a_{\mu}(A)$ and $-\Delta a_{\mu}\left(\varphi^{0}\right)$ as a function of $M_{A}=m_{\varphi^{0}}$ by fixing $t_{\beta}=100 . \Delta a_{\mu}$ increases rapidly with decreasing scalar masses. Since the negative contributions of the $C P$-even $\varphi^{0}$ become severe with decreasing $m_{\varphi^{0}}$, the inverted scenario receives a stronger constraint.

\section{THEORETICAL AND EXPERIMENTAL CONSTRAINTS ON THE TYPE-X 2HDM}

\section{A. Scanning strategies in three steps}

For the comprehensively study of the type-X 2 HDM in light of the muon $g-2$, we perform the successive and cumulative scan of the model parameters in three steps.

Step I: (O We demand that the model explains $\Delta a_{\mu}^{\text {obs }}$ at $2 \sigma$.

Step II: Among the parameters that survive Step I, we impose the constraints from theoretical stabilities and electroweak precision data, as detailed below.

(1) Theoretical stabilities [99,103,104]

(a) Higgs potential being bounded from below [103];

(b) Unitarity of scalar-scalar scatterings $[90,105]$; 
(c) Perturbativity [99];

(d) Vacuum stability [104].

(2) Peskin-Takeuchi electroweak oblique parameters [106].

We take the current best-fit results of [107]

$$
\begin{aligned}
S & =-0.01 \pm 0.10, \quad T=0.03 \pm 0.12, \\
U & =0.02 \pm 0.11, \\
\rho_{\mathrm{ST}} & =0.92, \quad \rho_{\mathrm{SU}}=-0.80, \quad \rho_{\mathrm{TU}}=-0.93,
\end{aligned}
$$

where $\rho_{i j}$ is the correlation matrix. The expressions of the contributions from the scalar boson loops to $S, T$, and $U$ are referred to Ref. [108,109]. We require $\Delta \chi^{2}\left(=\chi^{2}-\chi_{\min }^{2}\right)<7.81$.

Step III: For the parameters that survive Step II, we demand to satisfy the collider bounds.

(1) Higgs precision data by using HIGGSSIGNALS [110,111]:

The HIGGSSIGNALS-v2.2.0 [111] provides the $\chi^{2}$ value for 107 Higgs observables. Since our model has five parameters, the number of degrees of freedom for the $\chi^{2}$ analysis is 102 . We require that the calculated Higgs signal strengths be consistent with the experimental measurements at $2 \sigma$.

(2) Direct searches for new scalars at the LEP, Tevatron, and LHC:

We use the public code HIGGSBOUNDS [112]. Main search channels which affect the type-X 2HDM are

(a) LEP experiments:

(i) $e^{+} e^{-} \rightarrow Z \rightarrow A h \rightarrow \tau^{+} \tau^{-} \tau^{+} \tau^{-}$[113].

(b) LHC experiments:

(i) $h \rightarrow A A[114-119]$;

(ii) $H \rightarrow Z Z / W^{+} W^{-}[120-122]$;

(iii) $H \rightarrow h h[120,123-129]$;

(iv) $H / A \rightarrow \gamma \gamma[130,131], \tau^{+} \tau^{-}[132,133], \mu^{+} \mu^{-}$ [134-136], $b \bar{b}$ [137-139], $t \bar{t}$ [140];

(v) $A \rightarrow Z h[141,142]$; (vi) $A(H) \rightarrow Z H(A)[143,144]$;

(vii) $H^{ \pm} \rightarrow t b[145,146], \tau^{ \pm} \nu[147,148]$.

For each scattering process, we compute the $r_{95 \%}$ defined by

$$
r_{95 \%}=\frac{S_{2 \mathrm{HDM}}}{S_{\mathrm{obs}}^{95 \%}},
$$

where $S_{2 \mathrm{HDM}}\left(S_{\mathrm{obs}}^{95 \%}\right)$ is the predicted (observed) cross section. A point in the parameter space is excluded at the 95\% confidence level if $r_{95 \%}>1$.

In the normal scenario, we obtained $5 \times 10^{5}$ parameter sets that satisfy Step II. Step III excludes about $80 \%$ of the parameter sets that survived Step II. The exclusion is more severe in the inverted scenario, for which we separately collected $5 \times 10^{5}$ parameter sets that pass Step II. Only $\sim 1.8 \%$ parameter sets survive at Step III.

\section{B. Results in the normal scenario}

In Fig. 2 , we show the allowed $\left(M_{A}, t_{\beta}\right)$ at each step. The observed $\Delta a_{\mu}$ at Step I (left panel) demands $t_{\beta} \gtrsim 30$ and $M_{A} \lesssim 200 \mathrm{GeV}$, but does not limit the masses of $H$ and $H^{ \pm}$. Both $M_{H}$ and $M_{H^{ \pm}}$can reach about $1 \mathrm{TeV}$. At Step II (middle panel), a large cut on the parameter space is made, mainly on $M_{H}$ and $M_{H^{ \pm}}$. It is because the combination of the theoretical stability condition $\left(M_{A} \simeq M_{H^{ \pm}} \simeq M_{H} \approx M\right)$ and the intermediate $M_{A}$ at Step I lowers $M_{H}$ and $M_{H^{ \pm}}$. This feature is shown in Fig. 3 by the allowed $\left(M_{H}, M_{H^{ \pm}}\right)$ at Step III: Step II and Step III have similar results for $M_{H}$ and $M_{H^{ \pm}}$. There exist upper bounds of $M_{H} \lesssim 245 \mathrm{GeV}$ and $M_{H^{ \pm}} \lesssim 285 \mathrm{GeV}$. Besides, the correlation of $\Delta a_{\mu}$ with $M_{H}$ or $M_{H^{ \pm}}$is weak, as indicated by the mixed colors of $\Delta a_{\mu}$. As only the intermediate $M_{H}$ survives, the negative contribution of the $C P$-even $H$ to $\Delta a_{\mu}$ becomes significant.

Let us go back to discussing the allowed $\left(M_{A}, t_{\beta}\right)$. At Step III, which additionally imposes the constraints from the collider data at the LEP, Tevatron, and LHC, a large portion of the parameter space is removed: see the right
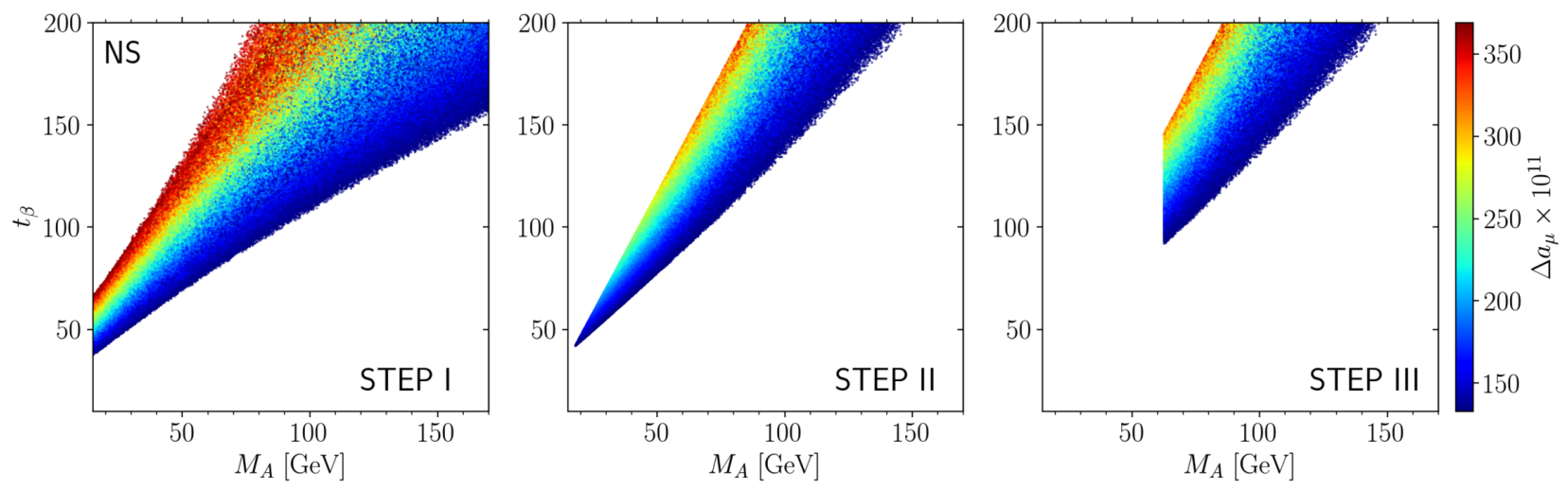

FIG. 2. In the normal scenario, the allowed parameter space of $\left(M_{A}, t_{\beta}\right)$ after Step I $\left(\Delta a_{\mu}^{\text {obs }}\right)$, Step II (Step I+Theory+EWPD), and Step III (Step II + Collider), with the color code indicating the value of $\Delta a_{\mu}$. 


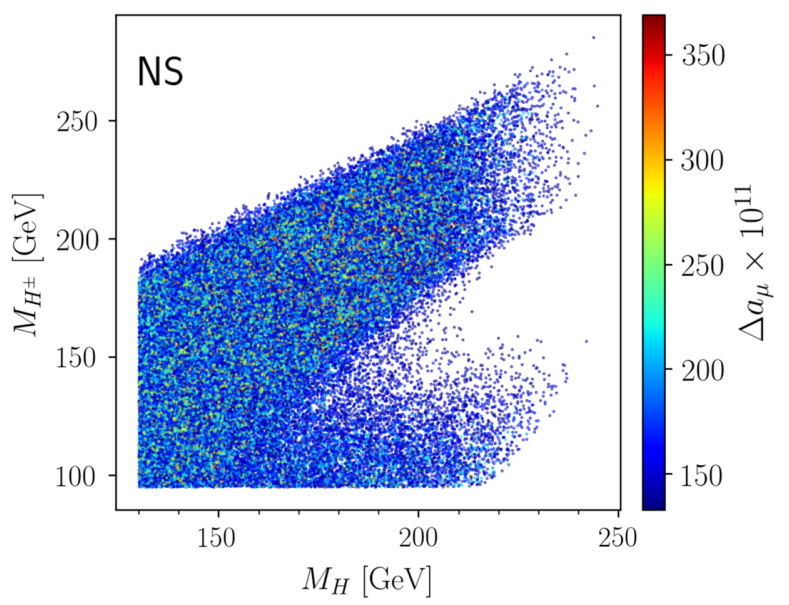

FIG. 3. The allowed $\left(M_{H}, M_{H^{ \pm}}\right)$in the normal scenario at Step III. The color code indicates the value of $\Delta a_{\mu}$.

panel in Fig. 2. We found that the recent LHC data play a crucial role in the curtailment. To demonstrate the role, we present the allowed parameter points in $\left(M_{A}, t_{\beta}\right)$ by the LHC data before 2015 (red) and those after 2015 (blue) in Fig. 4: 2015 is taken as the reference point in consideration of Ref. [78]. The new LHC data exclude the whole parameter space of $M_{A}<m_{125} / 2$ and $t_{\beta} \lesssim 90$. The accumulation of the LHC null results in the NP searches gives a significant implication on the type-X $2 \mathrm{HDM}$ in the context of muon $g-2$.

The question that follows is which LHC processes exclude the region of $M_{A}<m_{125} / 2$. In principle, multiple processes exclude one parameter set simultaneously. For efficient illustration, we present in Fig. 5 the smoking-gun process that has the largest deviation of the model

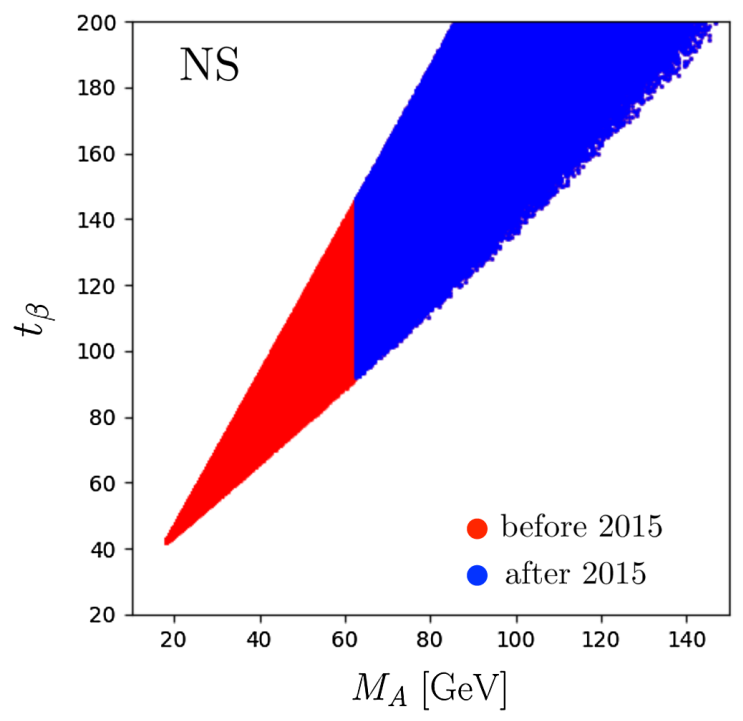

FIG. 4. In the normal scenario, the comparison of the allowed $\left(M_{A}, t_{\beta}\right)$ by using the LHC data before 2015 (red) and after 2015 (blue).

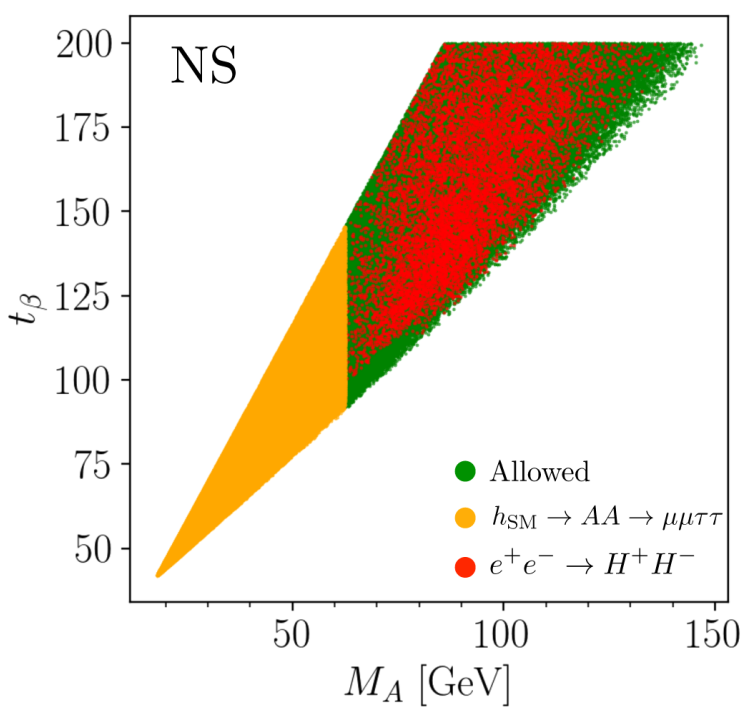

FIG. 5. In the normal scenario at Step III, the allowed points (green) and the excluded points (orange and red) in the parameter space of $\left(M_{A}, t_{\beta}\right)$. The orange points are excluded by $h_{\mathrm{SM}} \rightarrow$ $A A \rightarrow \mu^{+} \mu^{-} \tau^{+} \tau^{-}$at the LHC, and the red points are excluded by $e^{+} e^{-} \rightarrow H^{+} H^{-}$at the LEP.

prediction from the observation, $r_{95 \%}$ in Eq. (17). The green points pass all the constraints. The orange points are rejected by the LHC bounds on $h_{\mathrm{SM}} \rightarrow A A \rightarrow \mu^{+} \mu^{-} \tau^{+} \tau^{-}$ $[116,149]$. The red points are excluded by the combined LEP results of $e^{+} e^{-} \rightarrow H^{+} H^{-}$including the decays of $H^{+} H^{-}$into $c \bar{s} c \bar{s}, c \bar{s} \tau \nu, \tau \nu \tau \nu, W^{*} A \tau \nu$, and $W^{*} A W^{*} A$ [150]. The overlap of the allowed (green) and excluded (red) points is attributed to the projection of the five-dimensional hypervolume onto the two-dimensional $\left(M_{A}, t_{\beta}\right)$ plane. In summary, the normal scenario of the type-X $2 \mathrm{HDM}$ in light of the muon $g-2$ is phenomenologically viable for $t_{\beta} \gtrsim 90, M_{A} \in[62.5,145] \mathrm{GeV}, \quad M_{H} \in[130,245] \mathrm{GeV}$, and $M_{H^{ \pm}} \in[95,285] \mathrm{GeV}$.

\section{Results in the inverted scenario}

In the inverted scenario, the pattern of the exclusion at Step I, Step II, and Step III is similar to that in the normal scenario: see Fig. 6. In the quantitative aspect, however, there are some differences. At Step I, the observed $\Delta a_{\mu}$ prefers lighter $M_{A}$ than in the normal scenario, as the light $C P$-even $h$ makes a sizably negative contribution. The constraints at Step II are weaker than in the normal scenario. The perturbativity of $\lambda_{1}$, the most critical factor for the theoretical stability, is easier to satisfy with light $m_{h}$. At Step III (right panel), the collider constraints in the inverted scenario are stronger than in the normal scenario, leading to larger $t_{\beta}$ as $t_{\beta} \gtrsim 120$.

Figure 7 presents the collider smoking-gun processes in the inverted scenario. The green points are finally allowed. The orange and red points are excluded by $h_{\mathrm{SM}} \rightarrow A A$ $[116,149]$ and the LEP process $e^{+} e^{-} \rightarrow H^{+} H^{-}$[150], 

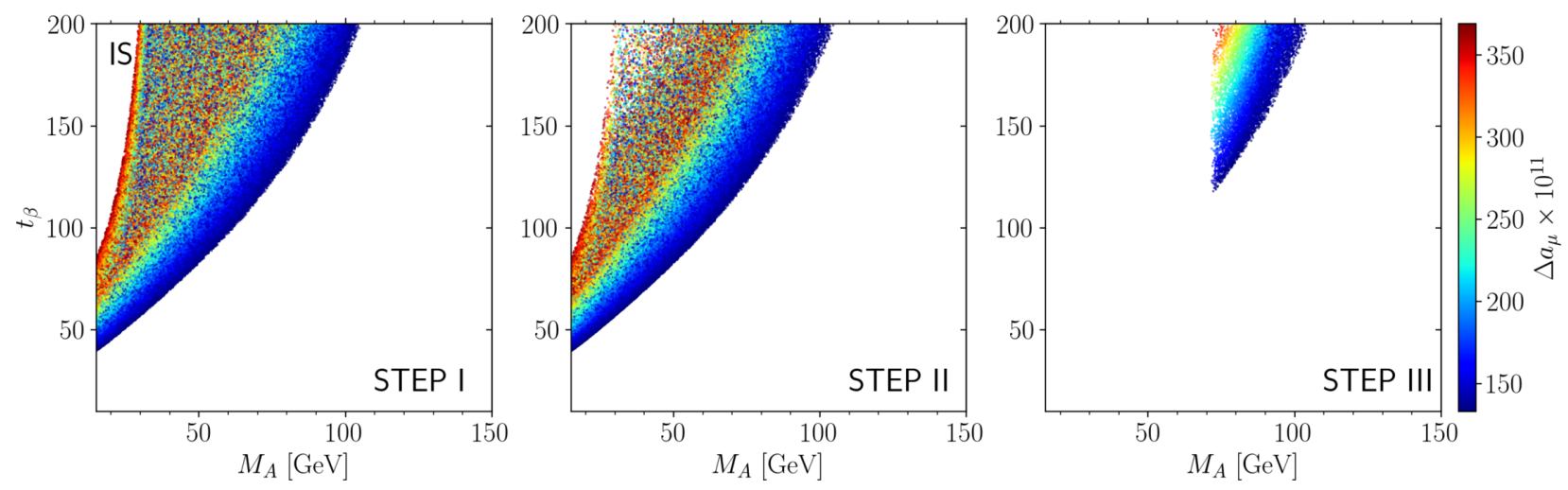

FIG. 6. In the inverted scenario, the allowed parameter space of $\left(M_{A}, t_{\beta}\right)$ at Step I, Step II, and Step III, with the color code indicating the value of $\Delta a_{\mu}$.

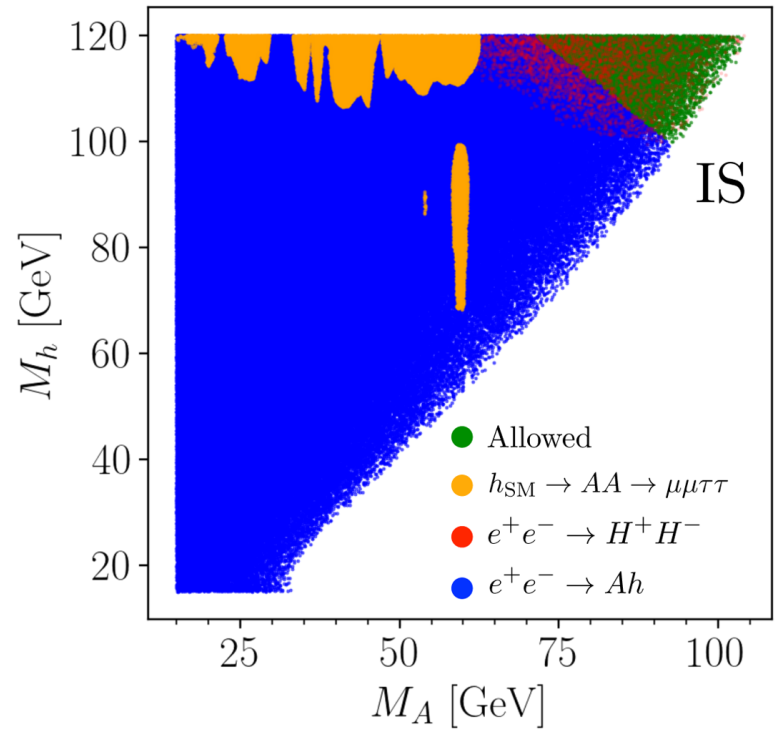

FIG. 7. In the inverted scenario at the final Step III, the allowed points (green) and the excluded points (orange, red, and blue). The orange points are excluded by $h_{\mathrm{SM}} \rightarrow A A \rightarrow \mu^{+} \mu^{-} \tau^{+} \tau^{-}$at the LHC, the red points by $e^{+} e^{-} \rightarrow H^{+} H^{-}$at the LEP, and the blue points by $e^{+} e^{-} \rightarrow A h$ at the LEP.

respectively. But the most vital role is played by the LEP process $e^{+} e^{-} \rightarrow Z^{*} \rightarrow A h$ [113] (blue points) because the $Z-A-h$ vertex, proportional to $c_{\beta-\alpha}$, is maximal in the alignment limit of the inverted scenario. We found that the constraint from $e^{+} e^{-} \rightarrow A h$ is so strong that only the kinematic ban of $\sqrt{s_{e e}}<M_{A}+M_{h}$ saves the parameter point.

In Fig. 8, we present the finally allowed $\left(M_{h}, M_{H^{ \pm}}\right)$in the inverted scenario with the color code indicating the value of $\Delta a_{\mu}$. As in the normal scenario, there is no correlation of $\Delta a_{\mu}$ with $M_{h}$ or $M_{H^{ \pm}}$: see the mixed color distribution. In summary, the inverted scenario survives for $t_{\beta} \gtrsim 120, \quad M_{A} \in[70,105] \mathrm{GeV}, \quad M_{H} \in[100,120] \mathrm{GeV}$, and $M_{H^{ \pm}} \in[95,185] \mathrm{GeV}$.

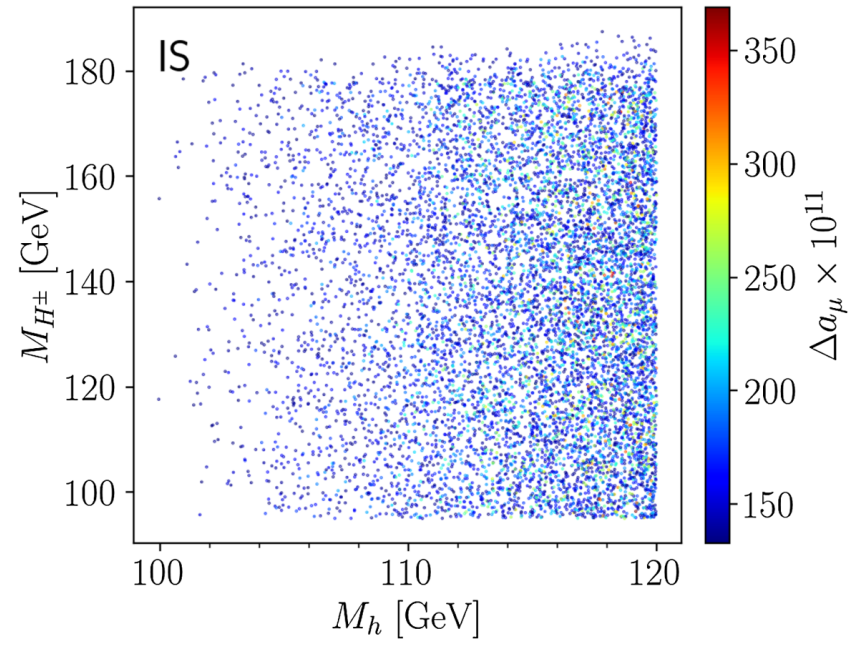

FIG. 8. The finally allowed $\left(M_{h}, M_{H^{ \pm}}\right)$in the inverted scenario with the color code indicating the value of $\Delta a_{\mu}$.

\section{IMPLICATIONS ON THE ELECTRON $g-2$ AND THE LHC COLLIDER SIGNATURES}

Upon obtaining the finally allowed parameter points of the type-X $2 \mathrm{HDM}$ in light of the new muon $g-2$, we investigate the phenomenological implications of the surviving parameters. First, we study the electron anomalous magnetic moment. For $\Delta a_{e}$, there is controversy over the value of the fine structure constant $\alpha$. Therefore, we check the consistency of the surviving parameters with $\Delta a_{e}$ rather than accept $\Delta a_{e}$ as an observable. Second, we study the LHC phenomenology so to suggest the golden mode for the hadrophobic scalar bosons. Since direct searches at high energy colliders provide independent information, the LHC exploration should continue.

\section{A. Electron anomalous magnetic moment}

As a flavor universal theory, type-X 2HDM has the same contributions to $\Delta a_{e}$ and $\Delta a_{\mu}$ except the differences of the electron and muon masses. Positive $\Delta a_{\mu}$ demands positive 

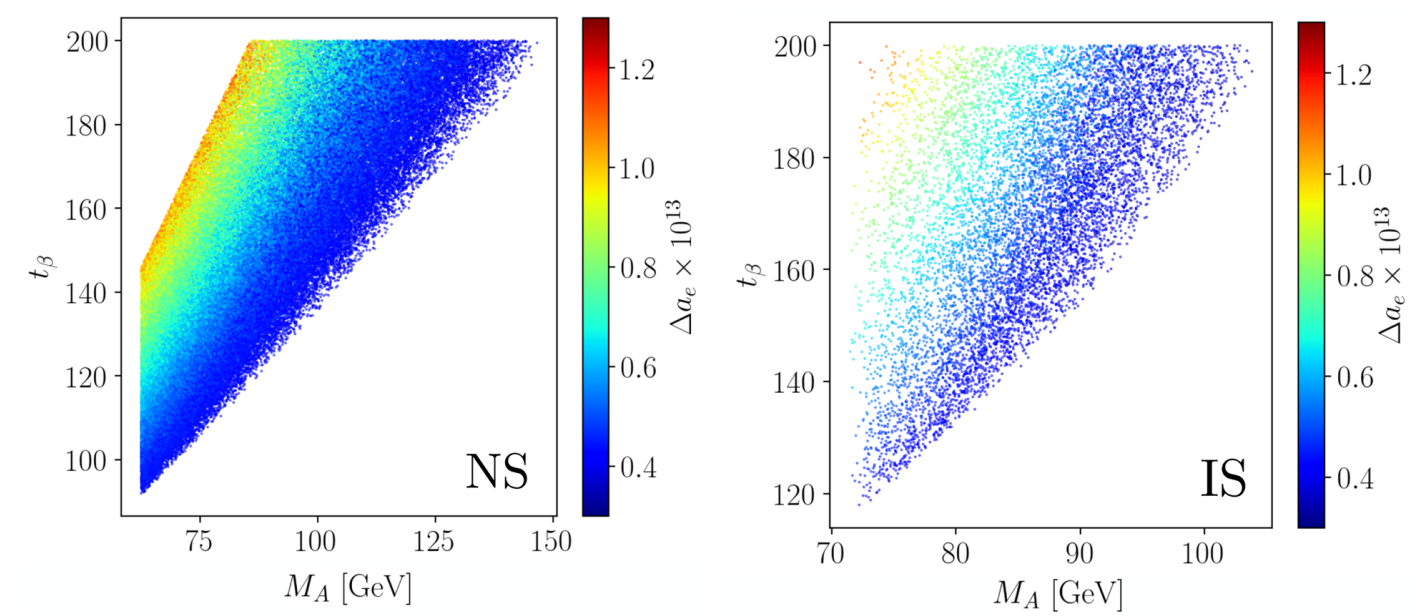

FIG. 9. $\Delta a_{e}$ of the finally allowed parameter points that satisfy all the theoretical and experimental constraints including $\Delta a_{\mu}$, projected on $\left(M_{A}, t_{\beta}\right)$. The left (right) panel corresponds to the normal (inverted) scenario.

$\Delta a_{e}$. In the measurement, however, $\Delta a_{e}$ has not been settled yet because of the discrepancy in the recent two experiments for the fine structure constant $\alpha$, the most sensitive input to $\Delta a_{e}$. Depending on whether we take the data from ${ }^{133} \mathrm{Cs}$ [86] or from ${ }^{87} \mathrm{Rb}$ [87], the deviations of the electron $g-2$ from the SM prediction [46,151,152] are substantially different as

$$
\begin{aligned}
& \Delta a_{e}^{\mathrm{Cs}}=-8.8(3.6) \times 10^{-13}, \\
& \Delta a_{e}^{\mathrm{Rb}}=4.8(3.0) \times 10^{-13} .
\end{aligned}
$$

At $2 \sigma$ level, $\Delta a_{e}^{\mathrm{Cs}}$ is negative while $\Delta a_{e}^{\mathrm{Rb}}$ can be positive.

In Fig. 9, we present the $\Delta a_{e}$ over the finally allowed $\left(M_{A}, t_{\beta}\right)$. The left (right) panel corresponds to the normal (inverted) scenario. The viable parameters predict $\Delta a_{e} \in$ $[0.36,1.17] \times 10^{-13}$ in the normal scenario and $\Delta a_{e} \in$ $[0.33,1.15] \times 10^{-13}$ in the inverted scenario. The $\Delta a_{e}^{\mathrm{Rb}}$ is explained at $2 \sigma$, except for the points along the upper-left boundary of the allowed $\left(M_{A}, t_{\beta}\right)$ space. The $\Delta a_{e}^{\mathrm{Cs}}$ is negative at $2 \sigma$, which is contradictory to the prediction of the model. At $3 \sigma$, however, it is consistent with the model.

\section{B. Production of the hadrophobic new scalars at the LHC}

For the LHC phenomenology, we point out two characteristics of the finally allowed parameters: (i) $t_{\beta}$ is extremely large; (ii) the masses of new scalar bosons are below about $300 \mathrm{GeV}$. The new scalar bosons with intermediate mass have escaped the LHC searches because of their hadrophobic nature due to large $t_{\beta}$. For the intermediate-mass $H^{ \pm}$, the current LHC search depends on its production via the decay of a top quark into $b H^{ \pm}$, followed by $H^{ \pm} \rightarrow \tau \nu[147,148]$. When $t_{\beta} \gtrsim 100$, however, the $H^{ \pm}-t-b$ vertex is extremely small, suppressing the production of the charged Higgs boson. For the intermediate-mass $A$ and $\varphi^{0}$, the LHC searches resort to
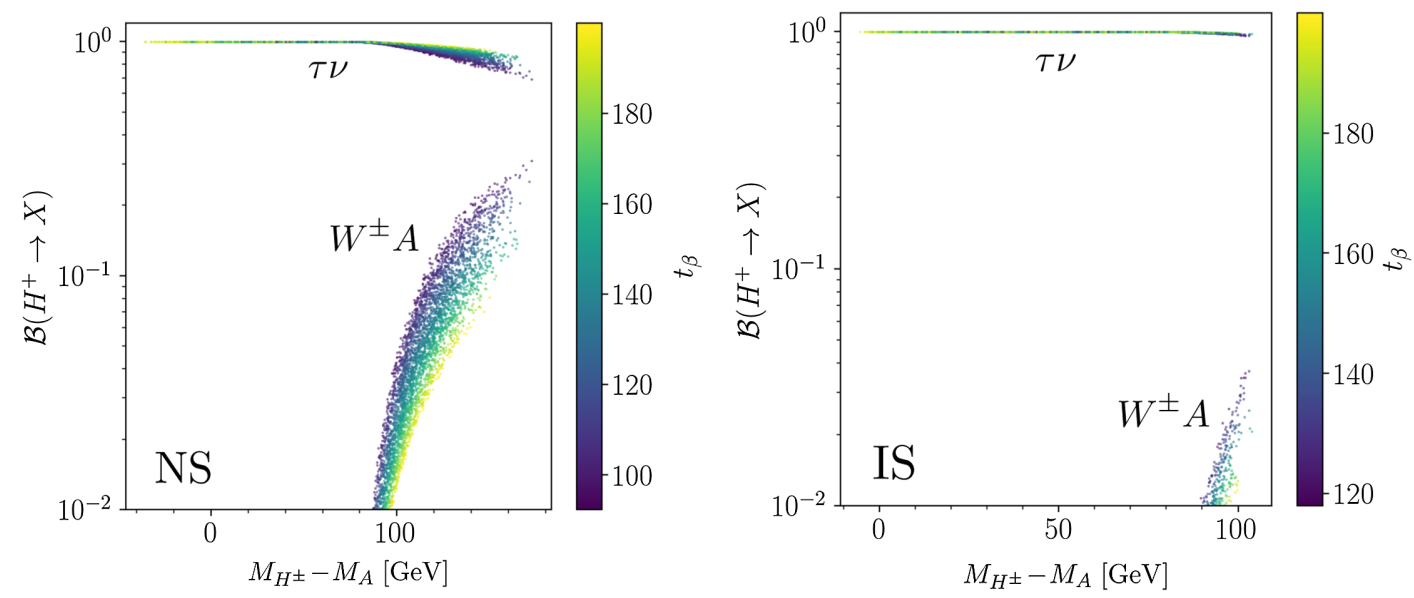

FIG. 10. The branching ratios of the charged Higgs boson as a function of $\left(M_{H^{ \pm}}-M_{A}\right)$ for the finally allowed parameter points. The left (right) panel corresponds to the normal (inverted) scenario. 

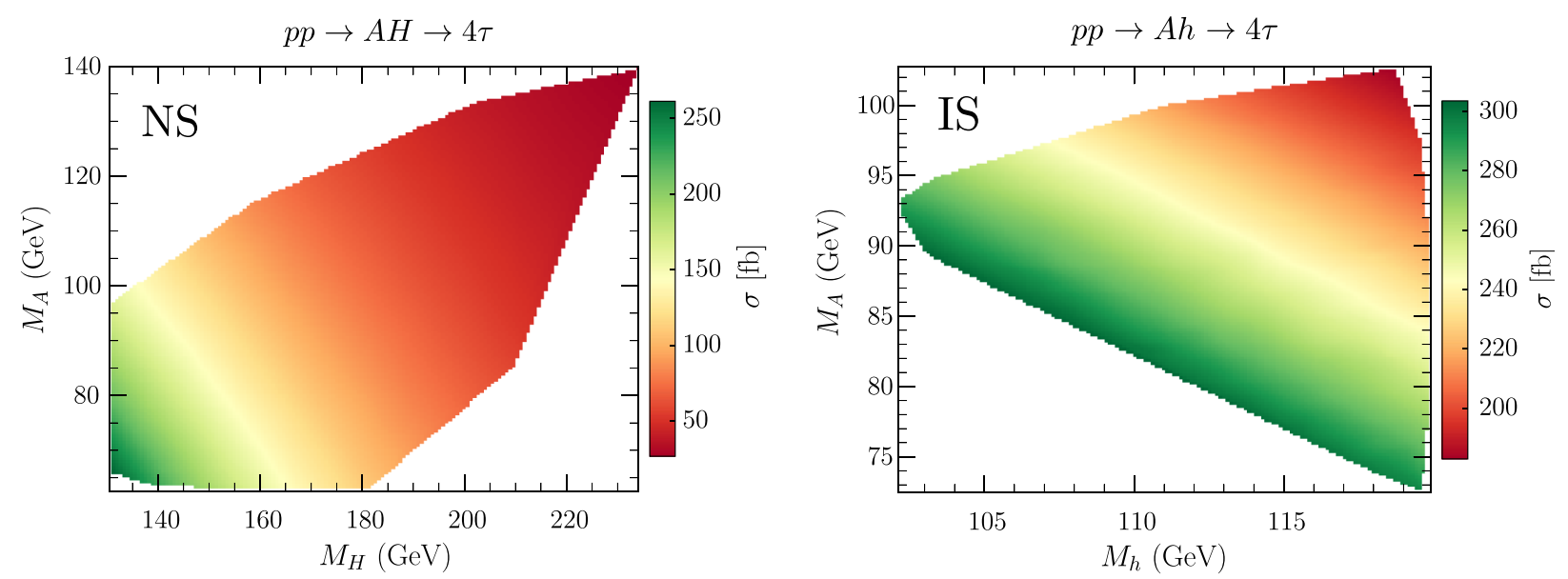

FIG. 11. The total cross section at the $14 \mathrm{TeV}$ LHC for the process $p p \rightarrow Z^{*} \rightarrow A H / A h \rightarrow 4 \tau$ of the finally allowed parameter points, projected on $\left(M_{H} / M_{h}, t_{\beta}\right)$. The left (right) panel corresponds to the normal (inverted) scenario.

the gluon fusion production via top quark loops, which is also suppressed. These hadrophobic new scalar bosons need different search strategies.
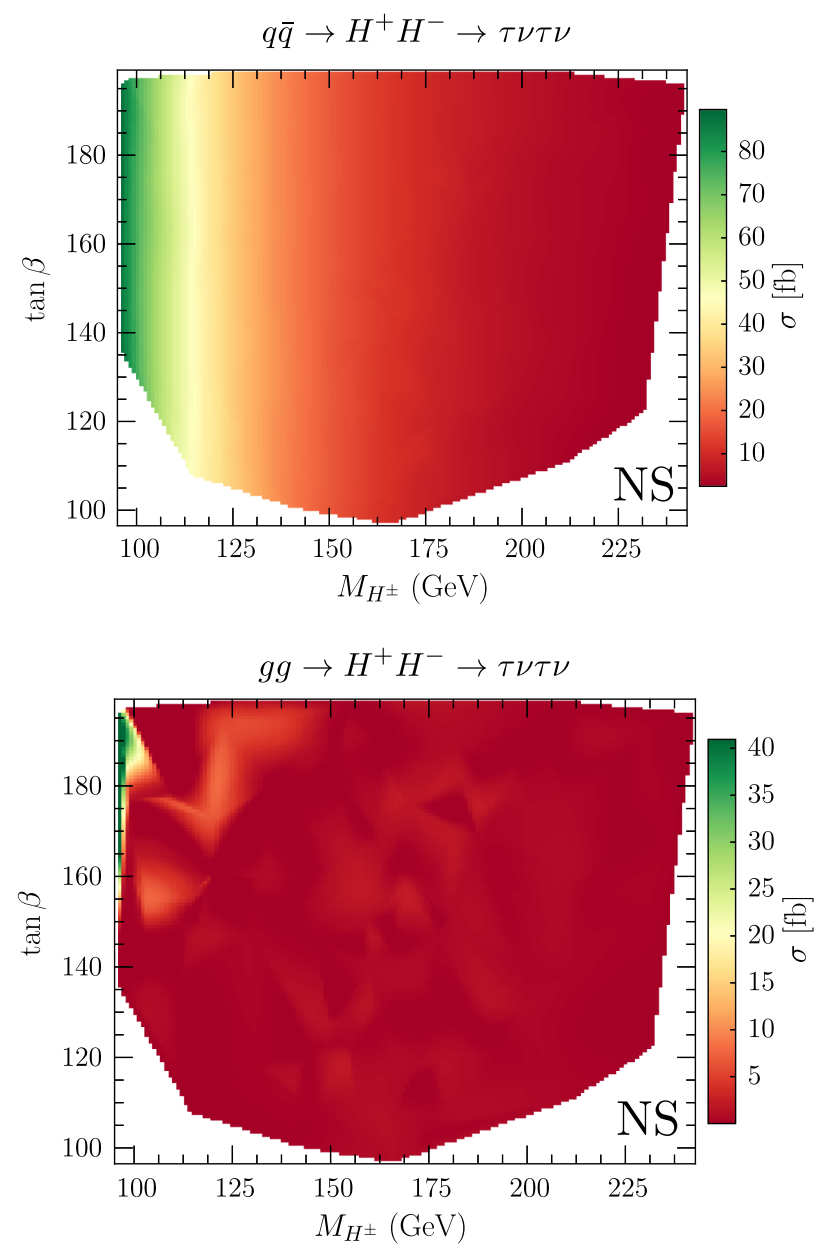

We study the branching ratios of $A, \varphi^{0}$, and $H^{ \pm}$in the viable parameter space. Both $A$ and $\varphi^{0}$ dominantly decay into $\tau^{+} \tau^{-}$. The branching ratios of $H \rightarrow Z A$ and
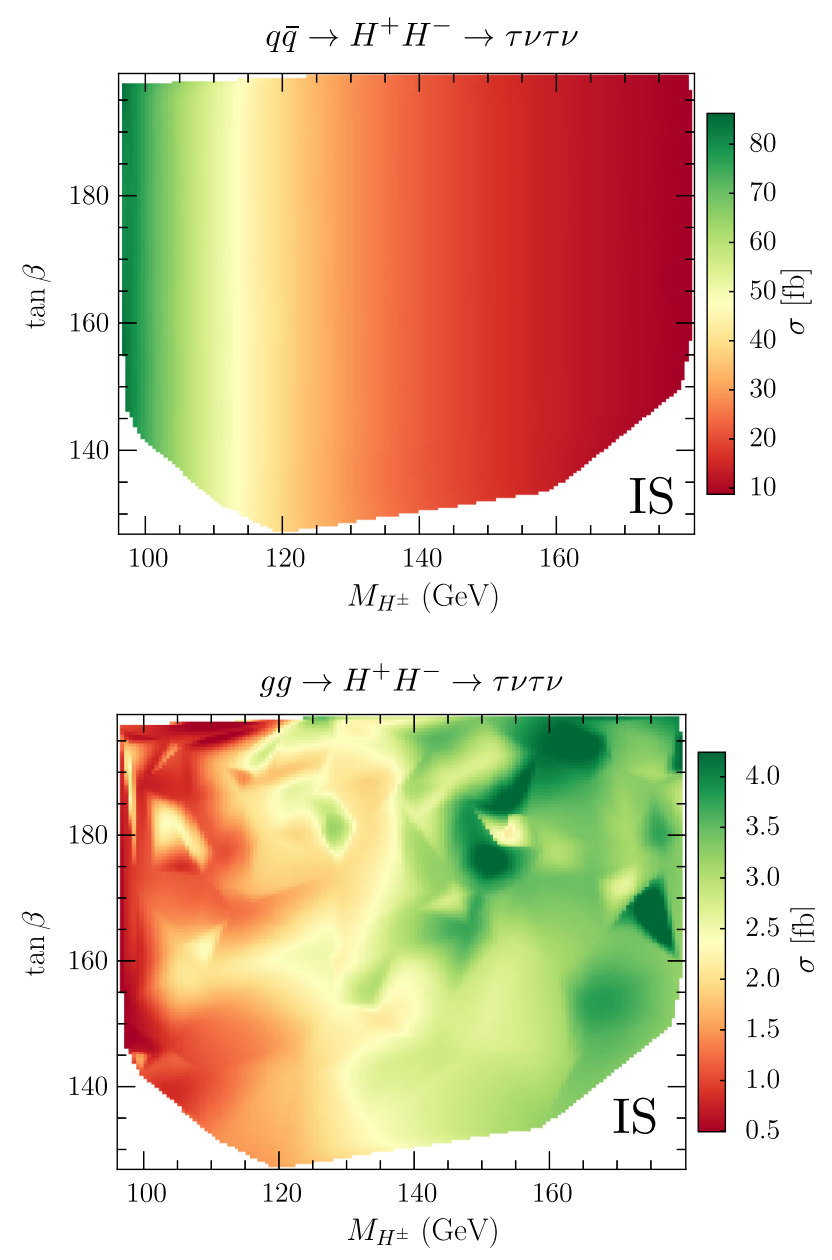

FIG. 12. The total cross section at the $14 \mathrm{TeV}$ LHC for the process $p p \rightarrow H^{+} H^{-} \rightarrow \tau \nu \tau \nu$ of the finally allowed parameter points, projected on $\left(M_{H^{ \pm}}, t_{\beta}\right)$. The results in the normal (inverted) scenario are in the left (right) panels. Two upper panels correspond to $q \bar{q} \rightarrow H^{+} H^{-}$, and two lower panels to the gluon fusion production. 
$H \rightarrow H^{ \pm} W^{\mp}$ are below $10 \%$. For the $H^{ \pm}$decays, Fig. 10 presents the scatter plot of the branching ratios as a function of $\left(M_{H^{ \pm}}-M_{A}\right)$ in the normal (left panel) and inverted (right panel) scenario. The color code indicates the value of $t_{\beta}$. The primary decay channel of $H^{ \pm}$is into $\tau^{ \pm} \nu$. The second important mode is $H^{ \pm} \rightarrow W^{ \pm} A$, which is sizable for larger $\left(M_{H^{ \pm}}-M_{A}\right)$ and smaller $t_{\beta}$. In the normal scenario, $\mathcal{B}\left(H^{ \pm} \rightarrow W^{ \pm} A\right)$ can reach up to about $30 \%$. In the inverted scenario, its maximum is only about $3 \%$.

Based on these characteristics, we consider the following two channels:

$$
\begin{gathered}
q \bar{q} \rightarrow Z^{*} \rightarrow A \varphi^{0} \rightarrow \tau^{+} \tau^{-} \tau^{+} \tau^{-}, \\
p p \rightarrow H^{+} H^{-} \rightarrow \tau^{+} \nu \tau^{-} \nu
\end{gathered}
$$

The process in Eq. (19) is efficient since the $Z-A-\varphi^{0}$ vertex has the maximal value in the alignment limit of both scenarios. In addition, $m_{\varphi^{0}}$ can be measured through the $\tau^{+} \tau^{-}$invariant mass distribution, differentiating the normal scenario from the inverted scenario. The pair production of charged Higgs bosons in Eq. (20) is almost uniquely determined by $M_{H^{ \pm}}$since the production is via the gauge couplings to $\gamma$ and $Z$.

Figure 11 shows the parton-level total cross sections of $p p \rightarrow Z^{*} \rightarrow A H / A h \rightarrow 4 \tau$ at the $14 \mathrm{TeV}$ LHC, by scanning all the viable parameter points. In both scenarios, we see a strong anti-correlation of $\sigma_{\text {tot }}$ with $M_{A}+M_{H}$. In the normal scenario (left panel), the total cross section lies between $\sim 25 \mathrm{fb}$ and $\sim 260 \mathrm{fb}$. In the inverted scenario, $\sigma_{\text {tot }}$ goes up to about $300 \mathrm{fb}$, larger than in the normal scenario. Considering the observed $\sigma(p p \rightarrow Z Z \rightarrow 4 \tau) \simeq 17 \mathrm{fb}$ at the $13 \mathrm{TeV}$ LHC [153,154], the process $p p \rightarrow A \varphi^{0} \rightarrow 4 \tau$ has a high potential to probe the model.

In Fig. 12, we present the prediction of the viable parameters to the total cross sections of the process $p p \rightarrow$ $H^{+} H^{-} \rightarrow \tau \nu \tau \nu$ at the $14 \mathrm{TeV}$ LHC. The results in the normal (inverted) scenario are in the left (right) panels. Two upper (lower) panels present the total cross sections for $q \bar{q} \rightarrow$ $H^{+} H^{-}\left(g g \rightarrow H^{+} H^{-}\right)$. In most parameter spaces, the DrellYan production has a much larger signal rate, since the hadrophobic nature of the charged Higgs boson suppresses the gluon fusion production mediated by the top quark loop. The irreducible backgrounds for the final state of $\tau^{+} \nu \tau^{-} \nu$ are $p p \rightarrow W^{+} W^{-} \rightarrow \tau^{+} \nu \tau^{-} \nu$ and $p p \rightarrow Z Z \rightarrow \tau^{+} \tau^{-} \nu \nu$. Considering $\sigma_{\text {tot }}^{\mathrm{SM}}\left(p p \rightarrow W^{+} W^{-} \rightarrow \tau^{+} \nu \tau^{-} \nu\right) \simeq 1.7 \mathrm{pb}$ [155] and $\sigma_{\text {tot }}^{\mathrm{SM}}\left(p p \rightarrow Z Z \rightarrow \tau^{+} \tau^{-} \nu \nu\right) \simeq 100 \mathrm{fb}[153,154]$ at the $13 \mathrm{TeV}$ LHC, there is a chance to see the process.

\section{LEPTON FLAVOR UNIVERSALITY DATA IN THE $\tau$ AND $Z$ DECAYS}

In Sec. IV, we found that the type-X $2 \mathrm{HDM}$ as a solution to the muon $g-2$ does not allow decoupling of any new Higgs boson. The generically flavor-universal model may yield excessive violation of the LFU in the $\tau$ and $Z$ decays, through the loop contributions mediated by new Higgs bosons. For the rigorous analysis, we first categorize the LFU data as follows:

(i) HFLAV global fit results in the $\tau$ decay: To parameterize the LFU in the $\tau$ decays, we introduce the coupling ratios defined by

$$
\begin{aligned}
& \left(\frac{g_{\tau}}{g_{\mu}}\right)^{2} \equiv \frac{\Gamma(\tau \rightarrow e \nu \nu)}{\Gamma(\mu \rightarrow e \nu \nu)} \times \frac{f\left(\rho_{\mu}^{e}\right)}{f\left(\rho_{\tau}^{e}\right)}, \\
& \left(\frac{g_{\tau}}{g_{e}}\right)^{2} \equiv \frac{\Gamma(\tau \rightarrow \mu \nu \nu)}{\Gamma(\mu \rightarrow e \nu \nu)} \times \frac{f\left(\rho_{\mu}^{e}\right)}{f\left(\rho_{\tau}^{\mu}\right)}, \\
& \left(\frac{g_{\mu}}{g_{e}}\right)^{2} \equiv \frac{\Gamma(\tau \rightarrow \mu \nu \nu)}{\Gamma(\tau \rightarrow e \nu \nu)} \times \frac{f\left(\rho_{\tau}^{e}\right)}{f\left(\rho_{\tau}^{\mu}\right)},
\end{aligned}
$$

where $\rho_{j}^{i}=m_{i}^{2} / m_{j}^{2}$ and

$$
f(x)=1-8 x+8 x^{3}-x^{4}-12 x^{2} \ln x .
$$

The second factors in the right-hand sides of Eq. (21) cancel the mass differences of the charged leptons. Including the hadronic decays of $\tau \rightarrow \pi \nu$ / $K \nu \rightarrow \mu \nu \nu$, we consider

$$
\begin{aligned}
& \mathcal{R}_{1}^{\tau} \equiv \frac{g_{\tau}}{g_{\mu}}, \quad \mathcal{R}_{2}^{\tau} \equiv \frac{g_{\tau}}{g_{e}}, \quad \mathcal{R}_{3}^{\tau} \equiv \frac{g_{\mu}}{g_{e}}, \\
& \mathcal{R}_{4}^{\tau} \equiv\left(\frac{g_{\tau}}{g_{\mu}}\right)_{\pi}, \quad \mathcal{R}_{5}^{\tau} \equiv\left(\frac{g_{\tau}}{g_{\mu}}\right)_{K},
\end{aligned}
$$

where $\left(\mathcal{R}_{1, \ldots 5}^{\tau}\right)^{\mathrm{SM}}=1$. Since $\mathcal{R}_{2}^{\tau} / \mathcal{R}_{1}^{\tau}=\mathcal{R}_{3}^{\tau}$, only four in Eq. (23) are independent. We should remove one redundant degree of freedom that has a zero eigenvalue in the covariance matrix.

(ii) Michel parameters: In the decay of $\tau^{-} \rightarrow \ell^{-} \nu \nu_{\tau}$, the energy and angular distribution of $\ell^{-}$provides valuable information on the LFU. The distribution is written in terms of the Michel parameters $\rho, \eta, \xi$, and $\delta$, as $[156,157]$

$$
\begin{aligned}
\frac{d^{2} \Gamma}{d x d \cos \theta^{*}} & \propto x^{2}\left[3(1-x)+\frac{2 \rho}{3}(4 x-3)+3 \eta x_{0} \frac{1-x}{x}\right. \\
& \left.+P_{\tau} \xi \cos \theta^{*}\left\{1-x+\frac{2 \delta}{3}(4 x-3)\right\}\right],
\end{aligned}
$$

where $x=2 E_{\ell} / m_{\tau}, x_{0}=2 m_{\ell} / m_{\tau}, P_{\tau}$ is the $\tau^{-}$ polarization, and $\theta^{*}$ is the angle between the $\ell^{-}$ momentum and the $\tau^{-}$spin quantization axis. In the SM [158], they are $^{3}$

\footnotetext{
${ }^{3}$ For the hadronic $\tau$ decay, only the $\xi_{h}$ was measured. In the literature, two conventions for the sign of $\xi_{h}$ coexist, $\xi_{h}>0$ in Refs. $[89,158,159]$ and $\xi_{h}<0$ in Refs. [80,160]. To unify with $\xi_{\ell}=1$ from the leptonic $\tau$ decays, we adopt the positive $\xi_{h}$ convention.
} 
$\rho^{\mathrm{SM}}=\frac{3}{4}, \quad \eta^{\mathrm{SM}}=0, \quad \xi^{\mathrm{SM}}=1, \quad(\xi \delta)^{\mathrm{SM}}=\frac{3}{4}$.

Including the leptonic and hadronic decays of $\tau^{-}$, we consider the Michel parameters of

$$
\begin{aligned}
& \mathcal{R}_{1}^{\mathrm{M}} \equiv \rho_{e}, \quad \mathcal{R}_{2}^{\mathrm{M}} \equiv(\xi \delta)_{e}, \quad \mathcal{R}_{3}^{\mathrm{M}} \equiv \xi_{e}, \\
& \mathcal{R}_{4}^{\mathrm{M}} \equiv \eta_{\mu}, \quad \mathcal{R}_{5}^{\mathrm{M}} \equiv \rho_{\mu}, \quad \mathcal{R}_{6}^{\mathrm{M}} \equiv(\xi \delta)_{\mu}, \quad \mathcal{R}_{7}^{\mathrm{M}} \equiv \xi_{\mu}, \\
& \mathcal{R}_{8}^{\mathrm{M}} \equiv \xi_{\pi}, \quad \mathcal{R}_{9}^{\mathrm{M}} \equiv \xi_{\rho}, \quad \mathcal{R}_{10}^{\mathrm{M}} \equiv \xi_{a_{1}} .
\end{aligned}
$$

(iii) $L F U$ in the $Z$ decay: From the partial decay rates of the leptonic $Z$ decays, we take two ratios of [161]

$$
\mathcal{R}_{1}^{Z} \equiv \frac{\Gamma\left(Z \rightarrow \mu^{+} \mu^{-}\right)}{\Gamma\left(Z \rightarrow e^{+} e^{-}\right)}, \quad \mathcal{R}_{2}^{Z} \equiv \frac{\Gamma\left(Z \rightarrow \tau^{+} \tau^{-}\right)}{\Gamma\left(Z \rightarrow e^{+} e^{-}\right)} .
$$

where $\left(\mathcal{R}_{1,2}^{Z}\right)^{\mathrm{SM}}=1$ if neglecting $m_{\tau}^{2} / m_{Z}^{2}$.

The type-X $2 \mathrm{HDM}$ makes two sorts of contributions to the observables in the $\tau$ decays, the tree-level contributions (mediated by the charged Higgs boson) and the one-loop level contributions. We parametrize them by

$$
\begin{aligned}
\delta_{\text {tree }} & =\frac{m_{\mu} m_{\tau} t_{\beta}^{2}}{M_{H^{ \pm}}^{2}}, \\
\delta_{\text {loop }} & =\frac{1}{16 \pi^{2}} \frac{m_{\tau}^{2} t_{\beta}^{2}}{v^{2}}\left[1+\frac{1}{4}\left\{H\left(\rho_{H^{ \pm}}^{A}\right)+H\left(\rho_{H^{ \pm}}^{\varphi^{0}}\right)\right\}\right],
\end{aligned}
$$

where $\rho_{j}^{i}=m_{i}^{2} / m_{j}^{2}$, and $H(x)=(1+x) \ln x /(1-x)$. If $M_{A}=M_{H}=M_{H^{ \pm}}$, we have $\delta_{\text {loop }}=0$ since $\lim _{x \rightarrow 1} H(x)=-2$. Therefore, similar masses of $H, A$, and $H^{ \pm}$in the viable parameter space help to suppress the loop-corrections to the $\tau$ decays.

For the HFLAV results, $\mathcal{R}_{i}^{\tau}$ 's in Eq. (23) receive new contributions, given by

$$
\begin{aligned}
& \mathcal{R}_{1}^{\tau}=\mathcal{R}_{4}^{\tau}=\mathcal{R}_{5}^{\tau}=1+\epsilon_{\text {loop }}^{\tau}, \\
& \mathcal{R}_{2}^{\tau}=1+\delta_{\text {loop }}+\epsilon_{\text {tree }}^{\tau}, \\
& \mathcal{R}_{3}^{\tau}=1+\epsilon_{\text {tree }}^{\tau}
\end{aligned}
$$

Here $\epsilon_{\text {tree }}^{\tau}$ is

$$
\epsilon_{\text {tree }}^{\tau}=\delta_{\text {tree }}\left[\frac{\delta_{\text {tree }}}{8}-\frac{m_{\mu}}{m_{\tau}} \frac{g\left(\rho_{\tau}^{\mu}\right)}{f\left(\rho_{\tau}^{\mu}\right)}\right],
$$

where $g(x)=1+9 x-9 x^{2}-x^{3}+6 x(1+x) \ln x$ and $f(x)$ is in Eq. (22).

For the Michel parameters, only the $\eta_{\mu},(\xi \delta)_{\mu}$, and $\xi_{\mu}$ are modified as

$$
\begin{aligned}
& \mathcal{R}_{4}^{\mathrm{M}} \equiv \eta_{\mu}=-\frac{2 \delta_{\text {tree }}\left(1+\delta_{\text {loop }}\right)}{4+\delta_{\text {tree }}^{2}} \\
& \mathcal{R}_{6}^{\mathrm{M}} \equiv(\xi \delta)_{\mu}=\frac{3}{4} \times \frac{4\left(1+\delta_{\text {loop }}\right)^{2}-\delta_{\text {tree }}^{2}}{4\left(1+\delta_{\text {loop }}\right)^{2}+\delta_{\text {tree }}^{2}} \\
& \mathcal{R}_{7}^{\mathrm{M}} \equiv \xi_{\mu}=\frac{4\left(1+\delta_{\text {loop }}\right)^{2}-\delta_{\text {tree }}^{2}}{4\left(1+\delta_{\text {loop }}\right)^{2}+\delta_{\text {tree }}^{2}}
\end{aligned}
$$

The corrections to $\rho_{e},(\xi \delta)_{e}$, and $\xi_{e}$ are suppressed by the small electron mass. For the hadronic $\tau$ decays, the corrections are independent of $t_{\beta}$, which is much smaller in the large $t_{\beta}$ limit than the $t_{\beta}^{2}$ corrections in the leptonic $\tau$ decays.

For $\mathcal{R}_{i}^{Z}$ 's, new contributions are written as

$$
\mathcal{R}_{i}^{Z}-1=\frac{2 g_{L}^{\mathrm{SM}} \operatorname{Re}\left(\delta g_{L}^{i}\right)+2 g_{R}^{\mathrm{SM}} \operatorname{Re}\left(\delta g_{R}^{i}\right)}{\left(g_{L}^{\mathrm{SM}}\right)^{2}+\left(g_{L}^{\mathrm{SM}}\right)^{2}}, \quad(i=\mu, \tau)
$$

where $g_{L}^{\mathrm{SM}}=s_{W}^{2}-1 / 2, g_{R}^{\mathrm{SM}}=s_{W}^{2}$, and the full expressions for $\delta g_{L / R}^{\mu, \tau}$ at one-loop level are referred to Ref. [80].

Rough estimation of new contributions is useful. Since $\delta_{\text {tree }} \gg \delta_{\text {loop }}$ and $\delta_{\text {tree }} \gg \epsilon_{\text {tree }}^{\tau}$, the dominant contribution is

$$
\eta_{\mu} \simeq-\frac{1}{2} \delta_{\text {tree }}
$$

While $\delta_{\text {tree }}$ is positive so that $\eta_{\mu}<0$ in the model, the ALEPH result is $\eta_{\mu}^{\mathrm{ALEPH}}=0.160 \pm 0.150$ [89]. The observed $\eta_{\mu}$ threatens the consistency of the type-X 2HDM with the LFU data.

Now we perform the global $\chi^{2}$ fit of the type-X 2 HDM to

$$
\Delta a_{\mu}, \quad \mathcal{R}_{1, \ldots, 5}^{\tau}, \quad \mathcal{R}_{1, \ldots, 10}^{\mathrm{M}}, \quad \mathcal{R}_{1,2}^{Z} .
$$

The experimental results of $\mathcal{R}$ 's and the correlation matrices are summarized in Appendix. Altogether we have 17 independent observables, $N_{\text {obs }}=17$, since we removed one redundant degree of freedom in $\mathcal{R}_{1, \ldots, 5}^{\tau}$. In the SM where the number of degree of freedom is $N_{\text {dof }}=17, \chi_{\text {min }}^{2}$ and $p$ value are

$$
\chi_{\min }^{2}(\mathrm{SM})=37.3, \quad p(\mathrm{SM})=0.003 .
$$

The $\Delta a_{\mu}^{\text {obs }}$ with the LFU data calls for NP.

For the type-X 2HDM, we address two issues. The first is the number of degrees of freedom, $N_{\text {dof }}=N_{\mathrm{obs}}-N_{\mathrm{par}}$, where $N_{\text {par }}$ is the number of free parameters. We subtract $N_{\text {par }}$ under the assumption that we use one free parameter to explain one observable. But our hypothesis model is not a free type-X $2 \mathrm{HDM}$. It is the model severely limited by the theoretical and experimental constraints. In favor of the type-X 2HDM, we take $N_{\text {dof }}=17$. The second issue is the 
range of the model parameters in the global $\chi^{2}$ fit. When finding $\chi_{\min }^{2}$, we may scan either the whole parameter space without imposing other constraints or only the parameter space consistent with all the constraints. In the two cases, $p$-values show big differences as follows:

$$
\begin{aligned}
p(\mathrm{NS}: \text { Step I }) & =0.58, \quad p(\mathrm{NS}: \text { Step III })=0.02, \\
p(\mathrm{IS}: \text { Step I }) & =0.059, \quad p(\text { IS : Step III })=0.02 .
\end{aligned}
$$

Without the LHC data, the type-X 2HDM in both scenarios well explains the $\Delta a_{\mu}$ and LFU data. With the combination of the LHC data and LFU data, however, the model is excluded as a solution to the new Fermilab measurement of the muon $g-2$.

\section{CONCLUSION}

In light of the recent measurement of the muon anomalous magnetic moment by Fermilab Muon $g-2$ experiment, we comprehensively study the type- $\mathrm{X}$ (lepton-specific) two Higgs doublet model (2HDM). Beyond explaining only the observed $\Delta a_{\mu}$, we included the theoretical stability conditions and almost all the available experimental results in the analysis. Since the Higgs precision data prefers the SM-like Higgs boson, more strongly for large $t_{\beta}$, we assumed the Higgs alignment. Two possible scenarios are studied, the normal scenario where the lighter $C P$-even $h$ becomes $h_{\mathrm{SM}}$ and the inverted scenario where the heavier $C P$-even $H$ is $h_{\mathrm{SM}}$. The model has five parameters, $m_{\varphi^{0}}, M_{A}, M_{H^{ \pm}}, M^{2}$, and $t_{\beta}$, where $\varphi^{0}=H$ in the normal scenario and $\varphi^{0}=h$ in the inverted scenario.

Various phenomenological conditions cause a chain reaction of constraining the model parameters. First, the large and positive $\Delta a_{\mu}^{\text {obs }}$ requires large $t_{\beta}$ and light $M_{A}$. The dominant contribution is from the $\tau^{ \pm}$loop mediated by $A$ in the two-loop Barr-Zee diagram. Unwanted is the negative contribution of $\varphi^{0}$ to the $\tau^{ \pm}$loop in the Barr-Zee diagram. But decoupling of $\varphi^{0}$ conflicts with the theoretical stability because of large $t_{\beta}$. The Higgs quartic coupling $\lambda_{1}$ has $t_{\beta}^{2}$ terms, which can easily break the perturbativity of $\lambda_{1}$. Requiring the $t_{\beta}^{2}$ terms to vanish yields $m_{\varphi^{0}}^{2} \approx M^{2}$. Perturbativity of other quartic couplings subsequently demands $M_{A} \simeq M_{H^{ \pm}} \simeq M \approx m_{\varphi^{0}}$. Decoupling of any new Higgs boson is not possible. The direct search bounds at the LEP and LHC exclude a large portion of the parameter space: $p p \rightarrow h_{\mathrm{SM}} \rightarrow A A$ in the normal scenario and $e^{+} e^{-} \rightarrow Z^{*} \rightarrow A h$ in the inverted scenario are the smoking signals. Only the region with $M_{A}>m_{h_{\mathrm{SM}}} / 2$ survives. In turn, $\Delta a_{\mu}^{\mathrm{obs}}$ demands $t_{\beta} \gtrsim 100$.

Through random scanning without any prior assumptions on the masses and couplings, we obtained the parameter points consistent with the muon $g-2$, theoretical stabilities, $S / T / U$ parameters, Higgs precision data, and direct search results. We also studied the phenomenological implications of the allowed parameter space. The model prediction to the electron anomalous magnetic moment is consistent with the observation, $\Delta a_{e}^{\mathrm{Cs}}$ (using the fine structure constant $\alpha$ from ${ }^{133} \mathrm{Cs}$ ) at $3 \sigma$, and $\Delta a_{e}^{\mathrm{Rb}}$ (using $\alpha$ from ${ }^{87} \mathrm{Rb}$ ) at $2 \sigma$. For the HL-LHC searches, we calculated the total cross sections for the hadrophobic new scalar bosons in two processes, $p p \rightarrow$ $A \varphi^{0} \rightarrow 4 \tau$ and $p p \rightarrow H^{+} H^{-} \rightarrow \tau \nu \tau \nu$. In particular, $p p \rightarrow$ $A \varphi^{0} \rightarrow 4 \tau$ has the total cross section around 25-260 fb in the normal scenario and $180-300 \mathrm{fb}$ in the inverted scenario. The model has a high potential to be probed at the LHC. As the final check of the model, we studied the lepton flavor universality in the $\tau$ and $Z$ decays. Through a global $\chi^{2}$ fit to $16 \mathrm{LFU}$ data and $\Delta a_{\mu}^{\mathrm{obs}}$, we showed that the combination of the LHC results and the LFU data excludes the type-X $2 \mathrm{HDM}$ as a solution to the muon $g-2$.

The confirmed deviation of the muon $g-2$ from the SM prediction by the recent Fermilab experiment indicates the dawn of a new physics era. The type-X 2HDM that explains $\Delta a_{\mu}$ is consistent with the LEP and LHC data in limited parameter space, but not with the LFU data in the $\tau$ and $Z$ decays. The future LHC searches targeting the specific parameters shall provide a valuable and independent probe of the model, which we strongly support.

\section{ACKNOWLEDGMENTS}

We would like to thank Kingman Cheung and Chih-Ting $\mathrm{Lu}$ for useful discussions. This work is supported by the National Research Foundation of Korea, Grant No. NRF2019R1A2C1009419.

\section{APPENDIX: USED PARAMETERS IN THE $\tau^{ \pm}$AND $Z$ DECAYS FOR THE GLOBAL $\chi^{2}$ ANALYSIS}

We present the experimental data on the parameters in the $\tau^{ \pm}$and $Z$ decays, which were used in the global $\chi^{2}$ analysis of Sec. VI.

(i) HFLAV global fit results in the $\tau$ decay: [88]

$$
\begin{aligned}
& \mathcal{R}_{1}^{\tau} \equiv\left(\frac{g_{\tau}}{g_{\mu}}\right)=1.0010 \pm 0.0014 \\
& \mathcal{R}_{2}^{\tau} \equiv\left(\frac{g_{\tau}}{g_{e}}\right)=1.0029 \pm 0.0014 \\
& \mathcal{R}_{3}^{\tau} \equiv\left(\frac{g_{\mu}}{g_{e}}\right)=1.0018 \pm 0.0014 \\
& \mathcal{R}_{4}^{\tau} \equiv\left(\frac{g_{\tau}}{g_{\mu}}\right)_{\pi}=0.9958 \pm 0.0026 \\
& \mathcal{R}_{5}^{\tau} \equiv\left(\frac{g_{\tau}}{g_{\mu}}\right)_{K}=0.9879 \pm 0.0063,
\end{aligned}
$$

and the correlation matrix for $\left(\mathcal{R}_{1}^{\tau}, \ldots, \mathcal{R}_{5}^{\tau}\right)$ is 


$$
\left(\boldsymbol{\rho}_{i j}^{\tau}\right)=\left(\begin{array}{rrrrr}
1.00 & 0.51 & -0.50 & 0.23 & 0.11 \\
0.51 & 1.00 & 0.49 & 0.25 & 0.10 \\
-0.50 & 0.49 & 1.00 & 0.02 & -0.01 \\
0.23 & 0.25 & 0.02 & 1.00 & 0.06 \\
0.11 & 0.10 & -0.01 & 0.06 & 1.00
\end{array}\right) .
$$

(ii) Michel parameters in the $\tau$ decay: [89]

$$
\begin{aligned}
& \mathcal{R}_{1}^{\mathrm{M}} \equiv \rho_{e}=0.747 \pm 0.019 \\
& \mathcal{R}_{2}^{\mathrm{M}} \equiv(\xi \delta)_{e}=0.788 \pm 0.066 \\
& \mathcal{R}_{3}^{\mathrm{M}} \equiv \xi_{e}=1.011 \pm 0.094 \\
& \mathcal{R}_{4}^{\mathrm{M}} \equiv \eta_{\mu}=0.160 \pm 0.150 \\
& \mathcal{R}_{5}^{\mathrm{M}} \equiv \rho_{\mu}=0.776 \pm 0.045 \\
& \mathcal{R}_{6}^{\mathrm{M}} \equiv(\xi \delta)_{\mu}=0.786 \pm 0.066 \\
& \mathcal{R}_{7}^{\mathrm{M}} \equiv \xi_{\mu}=1.030 \pm 0.120 \\
& \mathcal{R}_{8}^{\mathrm{M}} \equiv \xi_{\pi}=0.994 \pm 0.020 \\
& \mathcal{R}_{9}^{\mathrm{M}} \equiv \xi_{\rho}=0.987 \pm 0.012 \\
& \mathcal{R}_{10}^{\mathrm{M}} \equiv \xi_{a_{1}}=1.000 \pm 0.016
\end{aligned}
$$

and the correlation matrix for $\left(\mathcal{R}_{1}^{\mathrm{M}}, \ldots, \mathcal{R}_{10}^{\mathrm{M}}\right)$ is

$$
\left(\boldsymbol{\rho}_{i j}^{\mathrm{M}}\right)=\left(\begin{array}{rrrrrrrrrr}
1.00 & 0.00 & -0.23 & -0.02 & 0.02 & 0.00 & -0.02 & 0.10 & 0.00 & 0.00 \\
0.00 & 1.00 & 0.05 & 0.01 & 0.00 & 0.00 & -0.02 & -0.12 & 0.01 & 0.01 \\
-0.23 & 0.05 & 1.00 & 0.01 & 0.01 & -0.02 & -0.01 & -0.06 & -0.02 & -0.01 \\
-0.02 & 0.01 & 0.01 & 1.00 & 0.91 & 0.29 & 0.58 & 0.03 & -0.02 & -0.01 \\
0.02 & 0.00 & 0.01 & 0.91 & 1.00 & 0.25 & 0.45 & 0.06 & -0.02 & -0.01 \\
0.00 & 0.00 & -0.02 & 0.29 & 0.25 & 1.00 & 0.14 & -0.07 & -0.02 & -0.01 \\
-0.02 & -0.02 & -0.01 & 0.58 & 0.45 & 0.14 & 1.00 & -0.01 & -0.04 & -0.02 \\
0.10 & -0.12 & -0.06 & 0.03 & 0.06 & -0.07 & -0.01 & 1.00 & -0.28 & -0.20 \\
0.00 & 0.01 & -0.02 & -0.02 & -0.02 & -0.02 & -0.04 & -0.28 & 1.00 & -0.08 \\
0.00 & 0.01 & -0.01 & -0.01 & -0.01 & -0.01 & -0.02 & -0.20 & -0.08 & 1.00
\end{array}\right) .
$$

(iii) $L F U$ in the $Z$ decay: [161]

$$
\begin{aligned}
& \mathcal{R}_{1}^{Z} \equiv \frac{\Gamma\left(Z \rightarrow \mu^{+} \mu^{-}\right)}{\Gamma\left(Z \rightarrow e^{+} e^{-}\right)}=1.0009 \pm 0.0028, \\
& \mathcal{R}_{2}^{Z} \equiv \frac{\Gamma\left(Z \rightarrow \tau^{+} \tau^{-}\right)}{\Gamma\left(Z \rightarrow e^{+} e^{-}\right)}=1.0019 \pm 0.0032,
\end{aligned}
$$

where the correlation between $\mathcal{R}_{1}^{Z}$ and $\mathcal{R}_{2}^{Z}$ is +0.63 . 
[1] B. Abi et al. (Muon g-2 Collaboration), Measurement of the Positive Muon Anomalous Magnetic Moment to 0.46 ppm, Phys. Rev. Lett. 126, 141801 (2021).

[2] T. Albahri et al., Measurement of the anomalous precession frequency of the muon in the Fermilab Muon g-2 experiment, Phys. Rev. D 103, 072002 (2021).

[3] G. W. Bennett et al. (Muon g-2 Collaboration), Final report of the muon E821 anomalous magnetic moment measurement at BNL, Phys. Rev. D 73, 072003 (2006).

[4] T. Aoyama, M. Hayakawa, T. Kinoshita, and M. Nio, Complete Tenth-Order QED Contribution to the Muon g-2, Phys. Rev. Lett. 109, 111808 (2012).

[5] A. Czarnecki, W. J. Marciano, and A. Vainshtein, Refinements in electroweak contributions to the muon anomalous magnetic moment, Phys. Rev. D 67, 073006 (2003).

[6] C. Gnendiger, D. Stöckinger, and H. Stöckinger-Kim, The electroweak contributions to $(g-2)_{\mu}$ after the Higgs boson mass measurement, Phys. Rev. D 88, 053005 (2013).

[7] A. Kurz, T. Liu, P. Marquard, and M. Steinhauser, Hadronic contribution to the muon anomalous magnetic moment to next-to-next-to-leading order, Phys. Lett. B 734, 144 (2014).

[8] M. Davier, A. Hoecker, B. Malaescu, and Z. Zhang, Reevaluation of the hadronic vacuum polarisation contributions to the standard model predictions of the muon $g-2$ and $\alpha\left(m_{Z}^{2}\right)$ using newest hadronic cross-section data, Eur. Phys. J. C 77, 827 (2017).

[9] G. Colangelo, M. Hoferichter, and P. Stoffer, Two-pion contribution to hadronic vacuum polarization, J. High Energy Phys. 02 (2019) 006.

[10] A. Keshavarzi, D. Nomura, and T. Teubner, Muon $g-2$ and $\alpha\left(M_{Z}^{2}\right)$ : A new data-based analysis, Phys. Rev. D 97, 114025 (2018).

[11] A. Keshavarzi, D. Nomura, and T. Teubner, $g-2$ of charged leptons, $\alpha\left(M_{Z}^{2}\right)$, and the hyperfine splitting of muonium, Phys. Rev. D 101, 014029 (2020).

[12] M. Davier, A. Hoecker, B. Malaescu, and Z. Zhang, A new evaluation of the hadronic vacuum polarisation contributions to the muon anomalous magnetic moment and to $\boldsymbol{\alpha}\left(\mathbf{m}_{\mathbf{Z}}^{\mathbf{2}}\right)$, Eur. Phys. J. C 80, 241 (2020).

[13] B.-L. Hoid, M. Hoferichter, and B. Kubis, Hadronic vacuum polarization and vector-meson resonance parameters from $e^{+} e^{-} \rightarrow \pi^{0} \gamma$, Eur. Phys. J. C 80, 988 (2020).

[14] G. Colangelo, M. Hoferichter, and P. Stoffer, Constraints on the two-pion contribution to hadronic vacuum polarization, Phys. Lett. B 814, 136073 (2021).

[15] K. Melnikov and A. Vainshtein, Hadronic light-by-light scattering contribution to the muon anomalous magnetic moment revisited, Phys. Rev. D 70, 113006 (2004).

[16] G. Colangelo, M. Hoferichter, B. Kubis, M. Procura, and P. Stoffer, Towards a data-driven analysis of hadronic lightby-light scattering, Phys. Lett. B 738, 6 (2014).

[17] G. Colangelo, M. Hoferichter, A. Nyffeler, M. Passera, and P. Stoffer, Remarks on higher-order hadronic corrections to the muon $g-2$, Phys. Lett. B 735, 90 (2014).

[18] G. Colangelo, M. Hoferichter, M. Procura, and P. Stoffer, Dispersion relation for hadronic light-by-light scattering: Theoretical foundations, J. High Energy Phys. 09 (2015) 074.
[19] G. Colangelo, M. Hoferichter, M. Procura, and P. Stoffer, Dispersion relation for hadronic light-by-light scattering: Two-pion contributions, J. High Energy Phys. 04 (2017) 161.

[20] P. Masjuan and P. Sanchez-Puertas, Pseudoscalar-pole contribution to the $\left(g_{\mu}-2\right)$ : A rational approach, Phys. Rev. D 95, 054026 (2017).

[21] G. Colangelo, M. Hoferichter, M. Procura, and P. Stoffer, Rescattering Effects in the Hadronic-Light-by-Light Contribution to the Anomalous Magnetic Moment of the Muon, Phys. Rev. Lett. 118, 232001 (2017).

[22] M. Hoferichter, B.-L. Hoid, B. Kubis, S. Leupold, and S. P. Schneider, Pion-Pole Contribution to Hadronic Light-byLight Scattering in the Anomalous Magnetic Moment of the Muon, Phys. Rev. Lett. 121, 112002 (2018).

[23] M. Hoferichter, B.-L. Hoid, B. Kubis, S. Leupold, and S. P. Schneider, Dispersion relation for hadronic lightby-light scattering: Pion pole, J. High Energy Phys. 10 (2018) 141.

[24] G. Colangelo, F. Hagelstein, M. Hoferichter, L. Laub, and P. Stoffer, Short-distance constraints on hadronic light-bylight scattering in the anomalous magnetic moment of the muon, Phys. Rev. D 101, 051501 (2020).

[25] J. Bijnens, N. Hermansson-Truedsson, and A. RodríguezSánchez, Short-distance constraints for the HLbL contribution to the muon anomalous magnetic moment, Phys. Lett. B 798, 134994 (2019).

[26] T. Blum, N. Christ, M. Hayakawa, T. Izubuchi, L. Jin, C. Jung, and C. Lehner, Hadronic Light-by-Light Scattering Contribution to the Muon Anomalous Magnetic Moment from Lattice QCD, Phys. Rev. Lett. 124, 132002 (2020).

[27] J. Bijnens, N. Hermansson-Truedsson, L. Laub, and A. Rodríguez-Sánchez, Short-distance HLbL contributions to the muon anomalous magnetic moment beyond perturbation theory, J. High Energy Phys. 10 (2020) 203.

[28] S. Borsanyi et al., Leading hadronic contribution to the muon 2 magnetic moment from lattice QCD, arXiv:2002 .12347 .

[29] C. Lehner and A. S. Meyer, Consistency of hadronic vacuum polarization between lattice QCD and the R-ratio, Phys. Rev. D 101, 074515 (2020).

[30] A. Crivellin, M. Hoferichter, C. A. Manzari, and M. Montull, Hadronic Vacuum Polarization: $(g-2)_{\mu}$ versus Global Electroweak Fits, Phys. Rev. Lett. 125, 091801 (2020).

[31] A. Keshavarzi, W. J. Marciano, M. Passera, and A. Sirlin, Muon $g-2$ and $\Delta \alpha$ connection, Phys. Rev. D 102, 033002 (2020).

[32] B. Malaescu and M. Schott, Impact of correlations between $a_{\mu}$ and $\alpha_{\mathrm{QED}}$ on the EW fit, Eur. Phys. J. C 81, 46 (2021).

[33] A. Czarnecki and W. J. Marciano, The muon anomalous magnetic moment: A harbinger for 'new physics', Phys. Rev. D 64, 013014 (2001).

[34] H. Baer, V. Barger, and H. Serce, Anomalous muon magnetic moment, supersymmetry, naturalness, LHC search limits and the landscape, arXiv:2104.07597.

[35] A. Aboubrahim, M. Klasen, and P. Nath, What Fermilab $(g-2)_{\mu}$ experiment tells us about discovering SUSY at HL-LHC and HE-LHC, arXiv:2104.03839. 
[36] J. Cao, J. Lian, Y. Pan, D. Zhang, and P. Zhu, Imporved $(g-2)_{\mu}$ Measurement and singlino dark matter in the general NMSSM, arXiv:2104.03284.

[37] F. Wang, L. Wu, Y. Xiao, J. M. Yang, and Y. Zhang, GUTscale constrained SUSY in light of E989 muon g-2 measurement, arXiv:2104.03262.

[38] M. Van Beekveld, W. Beenakker, M. Schutten, and J. De Wit, Dark matter, fine-tuning and $(g-2)_{\mu}$ in the PMSSM, arXiv:2104.03245.

[39] M. Abdughani, Y.-Z. Fan, L. Feng, Y.-L. Sming Tsai, L. $\mathrm{Wu}$, and $\mathrm{Q}$. Yuan, A common origin of muon g-2 anomaly, Galaxy Center GeV excess and AMS-02 anti-proton excess in the NMSSM, arXiv:2104.03274.

[40] S. Baum, M. Carena, N. R. Shah, and C.E. M. Wagner, The tiny (g-2) muon wobble from Small $-\mu$ supersymmetry, arXiv:2104.03302.

[41] W. Ahmed, I. Khan, J. Li, T. Li, S. Raza, and W. Zhang, The natural explanation of the muon anomalous magnetic moment via the electroweak supersymmetry from the GmSUGRA in the MSSM, arXiv:2104.03491.

[42] H.-B. Zhang, C.-X. Liu, J.-L. Yang, and T.-F. Feng, Muon anomalous magnetic dipole moment in the $\mu \nu \mathrm{SSM}$, arXiv:2104.03489.

[43] M. Chakraborti, L. Roszkowski, and S. Trojanowski, GUT-constrained supersymmetry and dark matter in light of the new $(g-2)_{\mu}$ determination, arXiv:2104.04458.

[44] P. Athron, C. Balázs, D. H. Jacob, W. Kotlarski, D. Stöckinger, and H. Stöckinger-Kim, New physics explanations of $a_{\mu}$ in light of the FNAL muon $g-2$ measurement, arXiv:2104.03691.

[45] W. Yin, Muon $g-2$ anomaly in anomaly mediation, arXiv: 2104.03259.

[46] A. J. Buras, A. Crivellin, F. Kirk, C. A. Manzari, and M. Montull, Global analysis of leptophilic Z' bosons, arXiv:2104.07680.

[47] E. J. Chun and T. Mondal, Leptophilic bosons and muon g-2 at lepton colliders, arXiv:2104.03701.

[48] J. Liu, C. E. M. Wagner, and X.-P. Wang, A light complex scalar for the electron and muon anomalous magnetic moments, J. High Energy Phys. 03 (2019) 008.

[49] A. E. Cárcamo Hernández, S. Kovalenko, M. Maniatis, and I. Schmidt, Fermion mass hierarchy and g-2 anomalies in an extended 3HDM Model, arXiv:2104.07047.

[50] K. Ban, Y. Jho, Y. Kwon, S. C. Park, S. Park, and P.-Y. Tseng, A comprehensive study of vector leptoquark on the $B$-meson and muon g-2 anomalies, arXiv:2104.06656.

[51] M. Du, J. Liang, Z. Liu, and V.Q. Tran, A vector leptoquark interpretation of the muon $g-2$ and $B$ anomalies, arXiv:2104.05685.

[52] D. Borah, M. Dutta, S. Mahapatra, and N. Sahu, Muon $(g-2)$ and XENON1T excess with boosted dark matter in $L_{\mu}-L_{\tau}$ model, arXiv:2104.05656.

[53] L. Zu, X. Pan, L. Feng, Q. Yuan, and Y.-Z. Fan, Constraining $U(1)_{L_{\mu}-L_{\tau}}$ charged dark matter model for muon $g-2$ anomaly with AMS-02 electron and positron data, arXiv:2104.03340.

[54] J.-L. Yang, H.-B. Zhang, C.-X. Liu, X.-X. Dong, and T.-F. Feng, Muon $(g-2)$ in the B-LSSM, arXiv:2104.03542.

[55] A. Greljo, P. Stangl, and A. E. Thomsen, A model of muon anomalies, arXiv:2103.13991.
[56] B. Zhu and X. Liu, Probing light dark matter with scalar mediator: Muon $(g-2)$ deviation, the proton radius puzzle, arXiv:2104.03238.

[57] P. Escribano, J. Terol-Calvo, and A. Vicente, $(g-2)_{e, \mu}$ in an extended inverse type-III seesaw, arXiv:2104.03705.

[58] G. Arcadi, L. Calibbi, M. Fedele, and F. Mescia, Muon $g-2$ and $B$-anomalies from dark matter, arXiv:2104 .03228 .

[59] A. Crivellin and M. Hoferichter, Consequences of chirally enhanced explanations of $(g-2)_{\mu}$ for $h \rightarrow \mu \mu$ and $Z \rightarrow \mu \mu$, arXiv:2104.03202.

[60] M. A. Buen-Abad, J. Fan, M. Reece, and C. Sun, Challenges for an axion explanation of the muon $g-2$ measurement, arXiv:2104.03267.

[61] S.-F. Ge, X.-D. Ma, and P. Pasquini, Probing the dark axion portal with muon anomalous magnetic moment, arXiv:2104.03276.

[62] P. M. Ferreira, B. L. Gonçalves, F. R. Joaquim, and M. Sher, $(g-2)_{\mu}$ in the $2 \mathrm{HDM}$ and slightly beyond-an updated view, arXiv:2104.03367.

[63] X.-F. Han, T. Li, H.-X. Wang, L. Wang, and Y. Zhang, Lepton-specific inert two-Higgs-doublet model confronted with the new results for muon and electron g-2 anomalies and multi-lepton searches at the LHC, arXiv:2104.03227.

[64] C.-H. Chen, C.-W. Chiang, and T. Nomura, Muon $g-2$ in two-Higgs-doublet model with type-II seesaw mechanism, arXiv:2104.03275.

[65] N. Ghosh and J. Lahiri, Revisiting a generalized twoHiggs-doublet model in light of the muon anomaly and lepton flavor violating decays at the HL-LHC, Phys. Rev. D 103, 055009 (2021).

[66] N. Ghosh and J. Lahiri, Generalized 2HDM with wrongsign lepton Yukawa coupling, in light of $g_{\mu}-2$ and lepton flavor violation at the future LHC, arXiv:2103.10632.

[67] S.-P. Li, X.-Q. Li, Y.-Y. Li, Y.-D. Yang, and X. Zhang, Power-aligned 2HDM: A correlative perspective on $(g-2)_{e, \mu}$ J. High Energy Phys. 01 (2021) 034.

[68] F. J. Botella, F. Cornet-Gomez, and M. Nebot, Electron and muon $g-2$ anomalies in general flavour conserving two Higgs doublets models, Phys. Rev. D 102, 035023 (2020).

[69] S. Jana, V. P. K., and S. Saad, Resolving electron and muon $g-2$ within the 2HDM, Phys. Rev. D 101, 115037 (2020).

[70] S. Jana, P. K. Vishnu, W. Rodejohann, and S. Saad, Dark matter assisted lepton anomalous magnetic moments and neutrino masses, Phys. Rev. D 102, 075003 (2020).

[71] D. Anselmi, K. Kannike, C. Marzo, L. Marzola, A. Melis, K. Müürsepp et al., A fake doublet solution to the muon anomalous magnetic moment, arXiv:2104.03249.

[72] V. Keus, N. Koivunen, and K. Tuominen, Singlet scalar and 2HDM extensions of the standard model: $C P$-violation and constraints from $(g-2)_{\mu}$ and $e \mathrm{EDM}, \mathrm{J}$. High Energy Phys. 09 (2018) 059.

[73] D. Sabatta, A. S. Cornell, A. Goyal, M. Kumar, B. Mellado, and X. Ruan, Connecting muon anomalous magnetic moment and multi-lepton anomalies at LHC, Chin. Phys. C 44, 063103 (2020).

[74] K. Schmidt-Hoberg, F. Staub, and M. W. Winkler, Constraints on light mediators: Confronting dark matter searches with B physics, Phys. Lett. B 727, 506 (2013). 
[75] J. Cao, P. Wan, L. Wu, and J. M. Yang, Lepton-specific two-Higgs doublet model: Experimental constraints and implication on Higgs phenomenology, Phys. Rev. D 80, 071701 (2009).

[76] A. Broggio, E. J. Chun, M. Passera, K. M. Patel, and S. K. Vempati, Limiting two-Higgs-doublet models, J. High Energy Phys. 11 (2014) 058.

[77] L. Wang and X.-F. Han, A light pseudoscalar of 2HDM confronted with muon g-2 and experimental constraints, J. High Energy Phys. 05 (2015) 039.

[78] T. Abe, R. Sato, and K. Yagyu, Lepton-specific two Higgs doublet model as a solution of muon $g-2$ anomaly, J. High Energy Phys. 07 (2015) 064.

[79] E. J. Chun, S. Dwivedi, T. Mondal, and B. Mukhopadhyaya, Reconstructing a light pseudoscalar in the Type-X Two Higgs Doublet Model, Phys. Lett. B 774, 20 (2017).

[80] E. J. Chun and J. Kim, Leptonic precision test of leptophilic two-Higgs-doublet model, J. High Energy Phys. 07 (2016) 110.

[81] A. Cherchiglia, D. Stöckinger, and H. Stöckinger-Kim, Muon g-2 in the 2HDM: Maximum results and detailed phenomenology, Phys. Rev. D 98, 035001 (2018).

[82] L. Wang, J. M. Yang, M. Zhang, and Y. Zhang, Revisiting lepton-specific 2HDM in light of muon $g-2$ anomaly, Phys. Lett. B 788, 519 (2019).

[83] A. M. Sirunyan et al. (CMS Collaboration), Search for a standard model-like Higgs boson in the mass range between 70 and $110 \mathrm{GeV}$ in the diphoton final state in proton-proton collisions at $\sqrt{s}=8$ and $13 \mathrm{TeV}$, Phys. Lett. B 793, 320 (2019).

[84] S. M. Barr and A. Zee, Electric Dipole Moment of the Electron and of the Neutron, Phys. Rev. Lett. 65, 21 (1990).

[85] V. Ilisie, New Barr-Zee contributions to $(g-2)_{\mu}$ in twoHiggs-doublet models, J. High Energy Phys. 04 (2015) 077.

[86] R. H. Parker, C. Yu, W. Zhong, B. Estey, and H. Müller, Measurement of the fine-structure constant as a test of the standard model, Science 360, 191 (2018).

[87] L. Morel, Z. Yao, P. Cladé, and S. Guellati-Khélifa, Determination of the fine-structure constant with an accuracy of 81 parts per trillion, Nature (London) 588, 61 (2020).

[88] Y.S. Amhis et al. (HFLAV Collaboration), Averages of b-hadron, c-hadron, and $\tau$-lepton properties as of 2018 , Eur. Phys. J. C 81, 226 (2021).

[89] A. Heister et al. (ALEPH Collaboration), Measurement of the Michel parameters and the nu/tau helicity in tau lepton decays, Eur. Phys. J. C 22, 217 (2001).

[90] G. C. Branco, P. M. Ferreira, L. Lavoura, M. N. Rebelo, M. Sher, and J. P. Silva, Theory and phenomenology of twoHiggs-doublet models, Phys. Rep. 516, 1 (2012).

[91] S. L. Glashow and S. Weinberg, Natural conservation laws for neutral currents, Phys. Rev. D 15, 1958 (1977).

[92] E. A. Paschos, Diagonal neutral currents, Phys. Rev. D 15, 1966 (1977).

[93] M. Aoki, S. Kanemura, K. Tsumura, and K. Yagyu, Models of Yukawa interaction in the two Higgs doublet model, and their collider phenomenology, Phys. Rev. D 80, 015017 (2009).
[94] J. Song and Y.W. Yoon, $W \gamma$ decay of the elusive charged Higgs boson in the two-Higgs-doublet model with vectorlike fermions, Phys. Rev. D 100, 055006 (2019).

[95] ATLAS collaboration, A combination of measurements of Higgs boson production and decay using up to $139 \mathrm{fb}^{-1}$ of proton-proton collision data at $\sqrt{s}=13 \mathrm{TeV}$ collected with the ATLAS experiment, Report No. ATLAS-CONF2020-027, 2020, https://cds.cern.ch/record/2725733.

[96] M. Carena, I. Low, N. R. Shah, and C. E. M. Wagner, Impersonating the standard model Higgs boson: Alignment without decoupling, J. High Energy Phys. 04 (2014) 015 .

[97] A. Celis, V. Ilisie, and A. Pich, LHC constraints on twoHiggs doublet models, J. High Energy Phys. 07 (2013) 053.

[98] J. Bernon, J. F. Gunion, H. E. Haber, Y. Jiang, and S. Kraml, Scrutinizing the alignment limit in two-Higgsdoublet models: $m_{h}=125 \mathrm{GeV}$, Phys. Rev. D 92, 075004 (2015).

[99] S. Chang, S. K. Kang, J.-P. Lee, and J. Song, Higgs potential and hidden light Higgs scenario in two Higgs doublet models, Phys. Rev. D 92, 075023 (2015).

[100] D. Das and I. Saha, Search for a stable alignment limit in two-Higgs-doublet models, Phys. Rev. D 91, 095024 (2015).

[101] J. Bernon, J. F. Gunion, H. E. Haber, Y. Jiang, and S. Kraml, Scrutinizing the alignment limit in two-Higgsdoublet models. II. $m_{H}=125 \mathrm{GeV}$, Phys. Rev. D 93, 035027 (2016).

[102] G. Aad et al. (ATLAS Collaboration), Combined measurements of Higgs boson production and decay using up to $80 \mathrm{fb}^{-1}$ of proton-proton collision data at $\sqrt{s}=13 \mathrm{TeV}$ collected with the ATLAS experiment, Phys. Rev. D 101, 012002 (2020).

[103] I. P. Ivanov, Minkowski space structure of the Higgs potential in 2HDM, Phys. Rev. D 75, 035001 (2007).

[104] A. Barroso, P. M. Ferreira, I. P. Ivanov, and R. Santos, Metastability bounds on the two Higgs doublet model, J. High Energy Phys. 06 (2013) 045.

[105] A. Arhrib, Unitarity constraints on scalar parameters of the standard and two Higgs doublets model, in Workshop on Noncommutative Geometry, Superstrings and Particle Physics (2000) [arXiv:hep-ph/0012353].

[106] M.E. Peskin and T. Takeuchi, Estimation of oblique electroweak corrections, Phys. Rev. D 46, 381 (1992).

[107] P. A. Zyla et al. (Particle Data Group Collaboration), Review of particle physics, Prog. Theor. Exp. Phys. 2020, 083C01 (2020).

[108] H.-J. He, N. Polonsky, and S.-f. Su, Extra families, Higgs spectrum and oblique corrections, Phys. Rev. D 64, 053004 (2001).

[109] W. Grimus, L. Lavoura, O. M. Ogreid, and P. Osland, The Oblique parameters in multi-Higgs-doublet models, Nucl. Phys. B801, 81 (2008).

[110] P. Bechtle, S. Heinemeyer, O. Stål, T. Stefaniak, and G. Weiglein, Higgs Signals: Confronting arbitrary Higgs sectors with measurements at the Tevatron and the LHC, Eur. Phys. J. C 74, 2711 (2014). 
[111] P. Bechtle, S. Heinemeyer, T. Klingl, T. Stefaniak, G. Weiglein, and J. Wittbrodt, HiggsSignals-2: Probing new physics with precision Higgs measurements in the LHC 13 TeV era, Eur. Phys. J. C 81, 145 (2021).

[112] P. Bechtle, D. Dercks, S. Heinemeyer, T. Klingl, T. Stefaniak, G. Weiglein, and J. Wittbrodt, HiggsBounds-5: Testing Higgs sectors in the LHC 13 TeV era, Eur. Phys. J. C 80, 1211 (2020).

[113] S. Schael et al. (ALEPH, DELPHI, L3, OPAL, LEP Working Group for Higgs Boson Searches Collaborations), Search for neutral MSSM Higgs bosons at LEP, Eur. Phys. J. C 47, 547 (2006).

[114] M. Aaboud et al. (ATLAS Collaboration), Search for Higgs boson decays into a pair of light bosons in the $b b \mu \mu$ final state in $p p$ collision at $\sqrt{s}=13 \mathrm{TeV}$ with the ATLAS detector, Phys. Lett. B 790, 1 (2019).

[115] M. Aaboud et al. (ATLAS Collaboration), Search for the Higgs boson produced in association with a vector boson and decaying into two spin-zero particles in the $H \rightarrow$ $a a \rightarrow 4 b$ channel in $p p$ collisions at $\sqrt{s}=13 \mathrm{TeV}$ with the ATLAS detector, J. High Energy Phys. 10 (2018) 031.

[116] A. M. Sirunyan et al. (CMS Collaboration), Search for an exotic decay of the Higgs boson to a pair of light pseudoscalars in the final state of two muons and two $\tau$ leptons in proton-proton collisions at $\sqrt{s}=13 \mathrm{TeV}$, J. High Energy Phys. 11 (2018) 018.

[117] A. M. Sirunyan et al. (CMS Collaboration), Search for an exotic decay of the Higgs boson to a pair of light pseudoscalars in the final state with two muons and two b quarks in pp collisions at 13 TeV, Phys. Lett. B 795, 398 (2019).

[118] A. M. Sirunyan et al. (CMS Collaboration), Search for an exotic decay of the Higgs boson to a pair of light pseudoscalars in the final state with two $b$ quarks and two $\tau$ leptons in proton-proton collisions at $\sqrt{s}=13 \mathrm{TeV}$, Phys. Lett. B 785, 462 (2018).

[119] A. M. Sirunyan et al. (CMS Collaboration), Search for light pseudoscalar boson pairs produced from decays of the $125 \mathrm{GeV}$ Higgs boson in final states with two muons and two nearby tracks in pp collisions at $\sqrt{s}=13 \mathrm{TeV}$, Phys. Lett. B 800, 135087 (2020).

[120] M. Aaboud et al. (ATLAS Collaboration), Combination of searches for heavy resonances decaying into bosonic and leptonic final states using $36 \mathrm{fb}^{-1}$ of proton-proton collision data at $\sqrt{s}=13 \mathrm{TeV}$ with the ATLAS detector, Phys. Rev. D 98, 052008 (2018).

[121] A. M. Sirunyan et al. (CMS Collaboration), Search for a new scalar resonance decaying to a pair of $\mathrm{Z}$ bosons in proton-proton collisions at $\sqrt{s}=13 \mathrm{TeV}$, J. High Energy Phys. 06 (2018) 127.

[122] A. M. Sirunyan et al. (CMS Collaboration), Search for a heavy Higgs boson decaying to a pair of $\mathrm{W}$ bosons in proton-proton collisions at $\sqrt{s}=13 \mathrm{TeV}$, J. High Energy Phys. 03 (2020) 034.

[123] A. M. Sirunyan et al. (CMS Collaboration), Combination of Searches for Higgs Boson Pair Production in ProtonProton Collisions at $\sqrt{s}=13 \mathrm{TeV}$, Phys. Rev. Lett. 122, 121803 (2019).

[124] M. Aaboud et al. (ATLAS Collaboration), Search for Higgs boson pair production in the $\gamma \gamma W W^{*}$ channel using $p p$ collision data recorded at $\sqrt{s}=13 \mathrm{TeV}$ with the ATLAS detector, Eur. Phys. J. C 78, 1007 (2018).
[125] M. Aaboud et al. (ATLAS Collaboration), Search for pair production of Higgs bosons in the $b \bar{b} b \bar{b}$ final state using proton-proton collisions at $\sqrt{s}=13 \mathrm{TeV}$ with the ATLAS detector, J. High Energy Phys. 01 (2019) 030.

[126] M. Aaboud et al. (ATLAS Collaboration), Search for Higgs boson pair production in the $W W^{(*)} W W^{(*)}$ decay channel using ATLAS data recorded at $\sqrt{s}=13 \mathrm{TeV}$, J. High Energy Phys. 05 (2019) 124.

[127] M. Aaboud et al. (ATLAS Collaboration), Search for Resonant and Non-Resonant Higgs Boson Pair Production in the $b \bar{b} \tau^{+} \tau^{-}$Decay Channel in $p p$ Collisions at $\sqrt{s}=$ $13 \mathrm{TeV}$ with the ATLAS Detector, Phys. Rev. Lett. 121, 191801 (2018).

[128] G. Aad et al. (ATLAS Collaboration), Combination of searches for Higgs boson pairs in $p p$ collisions at $\sqrt{s}=$ $13 \mathrm{TeV}$ with the ATLAS detector, Phys. Lett. B 800, 135103 (2020).

[129] G. Aad et al. (ATLAS Collaboration), Search for the $H H \rightarrow b \bar{b} b \bar{b}$ process via vector-boson fusion production using proton-proton collisions at $\sqrt{s}=13 \mathrm{TeV}$ with the ATLAS detector, J. High Energy Phys. 07 (2020) 108.

[130] M. Aaboud et al. (ATLAS Collaboration), Search for new phenomena in high-mass diphoton final states using $37 \mathrm{fb}^{-1}$ of proton-proton collisions collected at $\sqrt{s}=13 \mathrm{TeV}$ with the ATLAS detector, Phys. Lett. B 775, 105 (2017).

[131] A. M. Sirunyan et al. (CMS Collaboration), Search for a standard model-like Higgs boson in the mass range between 70 and $110 \mathrm{GeV}$ in the diphoton final state in proton-proton collisions at $\sqrt{s}=8$ and $13 \mathrm{TeV}$, Phys. Lett. B 793, 320 (2019).

[132] A. M. Sirunyan et al. (CMS Collaboration), Search for additional neutral MSSM Higgs bosons in the $\tau \tau$ final state in proton-proton collisions at $\sqrt{s}=13 \mathrm{TeV}$, J. High Energy Phys. 09 (2018) 007.

[133] G. Aad et al. (ATLAS Collaboration), Search for Heavy Higgs Bosons Decaying into Two Tau Leptons with the ATLAS Detector Using $p p$ Collisions at $\sqrt{s}=13 \mathrm{TeV}$, Phys. Rev. Lett. 125, 051801 (2020).

[134] G. Aad et al. (ATLAS Collaboration), Search for highmass dilepton resonances using $139 \mathrm{fb}^{-1}$ of $p p$ collision data collected at $\sqrt{s}=13 \mathrm{TeV}$ with the ATLAS detector, Phys. Lett. B 796, 68 (2019).

[135] M. Aaboud et al. (ATLAS Collaboration), Search for scalar resonances decaying into $\mu^{+} \mu^{-}$in events with and without $b$-tagged jets produced in proton-proton collisions at $\sqrt{s}=$ $13 \mathrm{TeV}$ with the ATLAS detector, J. High Energy Phys. 07 (2019) 117.

[136] A. M. Sirunyan et al. (CMS Collaboration), Search for MSSM Higgs bosons decaying to $\mu^{+} \mu^{-}$in proton-proton collisions at $s=13 \mathrm{TeV}$, Phys. Lett. B 798, 134992 (2019).

[137] A. M. Sirunyan et al. (CMS Collaboration), Search for low-mass resonances decaying into bottom quark-antiquark pairs in proton-proton collisions at $\sqrt{s}=13 \mathrm{TeV}$, Phys. Rev. D 99, 012005 (2019).

[138] A. M. Sirunyan et al. (CMS Collaboration), Search for beyond the standard model Higgs bosons decaying into a $b \bar{b}$ pair in pp collisions at $\sqrt{s}=13 \mathrm{TeV}, \mathrm{J}$. High Energy Phys. 08 (2018) 113. 
[139] G. Aad et al. (ATLAS Collaboration), Search for heavy neutral Higgs bosons produced in association with $b$-quarks and decaying into $b$-quarks at $\sqrt{s}=13 \mathrm{TeV}$ with the ATLAS detector, Phys. Rev. D 102, 032004 (2020).

[140] A. M. Sirunyan et al. (CMS Collaboration), Search for heavy Higgs bosons decaying to a top quark pair in protonproton collisions at $\sqrt{s}=13 \mathrm{TeV}$, J. High Energy Phys. 04 (2020) 171.

[141] G. Aad et al. (ATLAS Collaboration), Search for a $C P$-odd Higgs boson decaying to $\mathrm{Zh}$ in pp collisions at $\sqrt{s}=8 \mathrm{TeV}$ with the ATLAS detector, Phys. Lett. B 744, 163 (2015).

[142] A. M. Sirunyan et al. (CMS Collaboration), Search for a heavy pseudoscalar boson decaying to a $\mathrm{Z}$ and a Higgs boson at $\sqrt{s}=13 \mathrm{TeV}$, Eur. Phys. J. C 79, 564 (2019).

[143] M. Aaboud et al. (ATLAS Collaboration), Search for a heavy Higgs boson decaying into a $Z$ boson and another heavy Higgs boson in the $\ell \ell b b$ final state in $p p$ collisions at $\sqrt{s}=13 \mathrm{TeV}$ with the ATLAS detector, Phys. Lett. B 783, 392 (2018).

[144] A. M. Sirunyan et al. (CMS Collaboration), Search for new neutral Higgs bosons through the $\mathrm{H} \rightarrow \mathrm{ZA} \rightarrow \ell^{+} \ell^{-} b \bar{b}$ process in pp collisions at $\sqrt{s}=13 \mathrm{TeV}$, J. High Energy Phys. 03 (2020) 055.

[145] M. Aaboud et al. (ATLAS Collaboration), Search for charged Higgs bosons decaying into top and bottom quarks at $\sqrt{s}=13 \mathrm{TeV}$ with the ATLAS detector, J. High Energy Phys. 11 (2018) 085.

[146] A. M. Sirunyan et al. (CMS Collaboration), Search for charged Higgs bosons decaying into a top and a bottom quark in the all-jet final state of $\mathrm{pp}$ collisions at $\sqrt{s}=13$ TeV, J. High Energy Phys. 07 (2020) 126.

[147] M. Aaboud et al. (ATLAS Collaboration), Search for charged Higgs bosons decaying via $H^{ \pm} \rightarrow \tau^{ \pm} \nu_{\tau}$ in the $\tau+$ jets and $\tau+$ lepton final states with $36 \mathrm{fb}^{-1}$ of $p p$ collision data recorded at $\sqrt{s}=13 \mathrm{TeV}$ with the ATLAS experiment, J. High Energy Phys. 09 (2018) 139.

[148] A. M. Sirunyan et al. (CMS Collaboration), Search for charged Higgs bosons in the $H^{ \pm} \rightarrow \tau^{ \pm} \nu_{\tau}$ decay channel in proton-proton collisions at $\sqrt{s}=13 \mathrm{TeV}$, J. High Energy Phys. 07 (2019) 142.

[149] V. Khachatryan et al. (CMS Collaboration), Search for light bosons in decays of the $125 \mathrm{GeV}$ Higgs boson in proton-proton collisions at $\sqrt{s}=8 \mathrm{TeV}$, J. High Energy Phys. 10 (2017) 076.

[150] G. Abbiendi et al. (ALEPH, DELPHI, L3, OPAL, LEP Collaborations), Search for charged Higgs bosons: Combined results using LEP data, Eur. Phys. J. C 73, 2463 (2013).

[151] T. Aoyama, T. Kinoshita, and M. Nio, Revised and improved value of the QED tenth-order electron anomalous magnetic moment, Phys. Rev. D 97, 036001 (2018).

[152] S. Laporta, High-precision calculation of the 4-loop contribution to the electron g-2 in QED, Phys. Lett. B 772, 232 (2017).

[153] M. Grazzini, S. Kallweit, and D. Rathlev, ZZ production at the LHC: Fiducial cross sections and distributions in NNLO QCD, Phys. Lett. B 750, 407 (2015).

[154] G. Aad et al. (ATLAS Collaboration), Measurement of the ZZ Production Cross Section in $p p$ Collisions at $\sqrt{s}=$ $13 \mathrm{TeV}$ with the ATLAS Detector, Phys. Rev. Lett. 116, 101801 (2016).

[155] M. Aaboud et al. (ATLAS Collaboration), Measurement of fiducial and differential $W^{+} W^{-}$production cross-sections at $\sqrt{s}=13 \mathrm{TeV}$ with the ATLAS detector, Eur. Phys. J. C 79, 884 (2019).

[156] L. Michel, Interaction between four half spin particles and the decay of the $\mu$ meson, Proc. Phys. Soc. London Sect. A 63, 514 (1950).

[157] H. E. Logan and D. MacLennan, Charged Higgs phenomenology in the lepton-specific two Higgs doublet model, Phys. Rev. D 79, 115022 (2009).

[158] A. Stahl, Michel parameters: Averages and interpretation, Nucl. Phys. B, Proc. Suppl. 76, 173 (1999).

[159] N. Shimizu et al. (Belle Collaboration), Measurement of the tau Michel parameters $\bar{\eta}$ and $\xi \kappa$ in the radiative leptonic decay $\tau^{-} \rightarrow \ell^{-} \nu_{\tau} \bar{\nu}_{\ell} \gamma$, Prog. Theor. Exp. Phys. 2018, 023C01 (2018).

[160] S. Gentile and M. Pohl, Physics of $\tau$ leptons, Phys. Rep. 274, 287 (1996).

[161] S. Schael et al. (ALEPH, DELPHI, L3, OPAL, SLD, LEP Electroweak Working Group, SLD Electroweak Group, SLD Heavy Flavour Group Collaborations), Precision electroweak measurements on the $Z$ resonance, Phys. Rep. 427, 257 (2006). 